\title{
Hydrogeochemical characteristics of the River Sava watershed in Slovenia
}

\author{
Hidrogeokemične značilnosti porečja reke Save v Sloveniji
}

\author{
Tjaša KANDUČ \& Nives OGRINC \\ Department of Environmental Sciences, Jožef Stefan Institute, Jamova 39, 1000 Ljubljana, Slovenia
}

Key words: River Sava, chemical composition, stable isotopes, sulphur; carbon, oxygen, deuterium terij

Ključne besede: reka Sava, kemijska sestava, stabilni izotopi, žveplo, ogljik, kisik, dev-

\begin{abstract}
The River Sava is a typical $\mathrm{HCO}_{3}{ }^{-}-\mathrm{Ca}^{2+}-\mathrm{Mg}^{2+}$ River. Total alkalinity increases in the part of the watershed composed of carbonate and clastic rocks, which are less resistant to weathering processes. $\mathrm{Ca}^{2+} / \mathrm{Mg}^{2+}$ ratios are around 2 in the carbonate part of the watershed and increase in the watershed composed of carbonate and clastic rocks, indicating dissolution of calcite with magnesium. According to PHREEQC for Windows calculations, the River Sava and its tributaries are oversaturated with respect to calcite and dolomite. $\delta^{18} \mathrm{O}_{\mathrm{H} 2 \mathrm{O}}$ and $\delta \mathrm{D}_{\mathrm{H} 2 \mathrm{O}}$ are related to the meteorological patterns in the drainage basin. River water temperatures fluctuate annually following air temperatures. The relationship between the temperature and $\delta^{18} \mathrm{O}_{\mathrm{H} 2 \mathrm{O}}$ and $\delta \mathrm{D}_{\mathrm{H} 2 \mathrm{O}}$ values primarily reflects the strong dependence of $\delta^{18} \mathrm{O}$ and $\delta \mathrm{D}$ on precipitation and evaporative enrichment in heavy oxygen and hydrogen isotopes of infiltrating water recharging the River Sava from its slopes. The $\delta^{13} \mathrm{C}_{\text {DIC }}$ values are controlled by processes in the terrestrial ecosystem and stream processes such as: (1) dissolution of carbonates, (2) soil derived $\mathrm{CO}_{2}$, and (3) equilibration with atmospheric $\mathrm{CO}_{2}$. Lower $\delta^{13} \mathrm{C}_{\mathrm{DIC}}$ values are observed in the spring sampling season due to abundant precipitation related to soil leaching of $\mathrm{CO}_{2}$ in the river system. From discharge and concentration measurements of sulphate and according to the drainage area of the River Sava basin, the annual sulphur flux at the border with Croatia was estimated to be $1.4 \times 10^{7} \mathrm{~g} \mathrm{SO}_{4} / \mathrm{km}^{2}$. Assuming that the sources of $\mathrm{SO}_{4}{ }^{2-}$ to the Sava are its tributaries, precipitation and other sources, the contributions of these inputs were calculated according to steady state equations and estimated to be $52: 8: 40 \%$, respectively. Other sources are attributed to human influences such as industrial pollution and oxidation of sulphides.
\end{abstract}

\section{Izvleček}

Reka Sava je tipična $\mathrm{HCO}_{3}^{-}-\mathrm{Ca}^{2+}-\mathrm{Mg}^{2+}$ reka. Totalna alkalnost narašča po porečju navzdol, ki ga sestavljajo poleg karbonatnih tudi klastične kamnine, ki so manj odporne na procese preperevanja. Razmerje $\mathrm{Ca}^{2+} / \mathrm{Mg}^{2+}$ je okrog $2 \mathrm{v}$ karbonatnem delu porečja in naraste v porečju, ki ga sestavljajo karbonatne in klastične kamnine, kar kaže na raztapljanje kalcita z magnezijem. Glede na izračune PHREEQC for Windows so reka Sava in njeni pritoki prenasičeni s kalcitom in dolomitom. $\delta^{18} \mathrm{O}_{\mathrm{H} 2 \mathrm{O}}$ in $\delta \mathrm{D}_{\mathrm{H} 2 \mathrm{O}}$ vrednosti sta povezani z meteorološkimi spremenljivkami v drenažnem bazenu. Temperatura rečne vode se letno spreminja glede na temperature v zraku. Povezava med temperaturo, $\delta^{18} \mathrm{O}_{\mathrm{H} 2 \mathrm{O}}$ odraža močno odvisnost $\delta^{18} \mathrm{O} v$ padavinah in evaporativno obogatitev na težjih izotopih kisika infiltrirajoče vode s pobočij, ki napaja reko Savo. $\delta^{13} \mathrm{C}_{\text {DIC }}$ vrednosti kontrolirajo procesi v terestričnih ekosistemih in v reki, kot so: (1) raztapljanje karbonatov, (2) preperinski $\mathrm{CO}_{2}$ ter (3) uravnoteženje $\mathrm{z}$ atmosferskim $\mathrm{CO}_{2}$. Bolj negativne vrednosti $\delta^{13} \mathrm{C}_{\mathrm{DIC}}$ so posledica večje količine padavin in spiranja izotopsko lažjega preperinskega $\mathrm{CO}_{2} \mathrm{~s}$ terestričnega ekosistema v spomladanskih mesecih. Iz podatkov o pretokih, koncentracijah sulfata v vodi in odvodnjevalni površini smo izračunali letni snovni tok žvepla na meji s Hrvaško, ki znaša $1,4 \times 10^{7} \mathrm{~g} \mathrm{SO}_{4} / \mathrm{km}^{2}$. Glede nato, da smo predpostavili sledeče vire sulfata v reki Savi: pritoke, padavine in ostale vire, smo izračunali deleže posameznih prispevkov iz ravnotežnih enačb koncentracijske in izotopske masne bilance, ki znašajo $52: 8: 40 \%$ Ostale vire lahko pripišemo industrijski onesnaženosti in oksidaciji sulfidnih mineralov. 


\section{Introduction}

The geochemical study of river water allows important information to be obtained on chemical weathering of rocks/soil and the chemical and isotopic compositions of the drainage basin (Gibbs, 1972; Reeder et al., 1972; Hu et al., 1982; Stallard \& Edmond, 1983; Goldstein \& Jacobsen, 1987; Elderfield et al., 1990; Zhang et al., 1995; Huh et al., 1998). Since carbonate weathering largely dominates the water chemistry of river waters, characterization of the water chemistry of rivers draining carbonate-dominated terrain is crucial to precisely identify the various contributions of the different sources to water solutes, and to estimate weathering rates of the continental crust and associated $\mathrm{CO}_{2}$ consumption (Fairchild et al., 1994, 1999, 2000; Gaillardet et al., 1999; Liu \& Zhao, 2000).

Rivers also reflect biogeochemical processes occurring in their catchment areas and help to quantify material transport from land to ocean (Palmer et al., 2001). Within this context, understanding of the carbon cycle is particulary important because it helps to evaluate the health of the river and its catchment basin (Telmer \& Veizer, 1999). Investigations of major elements and stable carbon isotopes of dissolved inorganic carbon $\left(\delta^{13} \mathrm{C}_{\mathrm{DIC}}\right)$ are useful for such studies and to evaluate environmental influences $(\mathrm{Ka}$ rim \& Veizer, 2000; Barth et al., 2003).

Rivers represent a linkage between precipitation and groundwater, which all together form the hydrogeological cycle. Stable isotopes of oxygen and hydrogen are used to trace processes of evaporation, condensation, snow melting, mixing of waters of different origin and recharge conditions in the studied watersheds (Clark \& Fritz, 1997).

The natural sources of $\mathrm{SO}_{4}{ }^{2-}$ in river water are rainfall, groundwater, and weathering of S-rich minerals. Human activities (e.g. air pollution, mining, smelting of sulphide ore, refining of petroleum, and chemical industries) also contribute to the $\mathrm{SO}_{4}{ }^{2-}$ content of river water. These multiple sources of sulphate can frequently be distinguished by their specific isotopic signatures. For example, river sulphate derived from dissolution of evaporates has positive $\delta^{34} \mathrm{~S}$ values whereas sulphate from oxidation of sulphides or from biogenic emissions may have strongly negative $\delta^{34} \mathrm{~S}$. The latter is due to the fact that sulphide minerals tend to be isotopically lighter than sulphate minerals, and hence the oxidation of sulphides (and that of organic sulphur) transposes the depleted $\delta^{34} \mathrm{~S}$ signal to sulphate products (Pearson \& Rightmire, 1980).

The main objectives of this study were to understand the hydrogeochemical characteristics of the River Sava watershed through time (different seasons and flow regimes), and to quantify sources, fluxes and sinks of carbon and sulphur from the isotopic characteristics of their dissolved species.

\section{Characterization of the River Sava drainage basin}

The River Sava, the largest river in Slovenia and a tributary of the Danube, originates in the Triassic carbonate hinterland at Zelenci (Figure 1, location 1) as the Sava Dolinka and the karst spring Savica (Figure 1 , location 6) as the Sava Bohinjka. The confluence of these two sources is at Radovljica. From there the river is named Sava and finishes its course at Belgrade, merging with the Danube. From the source of the Sava Dolinka to the national border with Croatia its length is $219 \mathrm{~km}$. Along its studied course of $219 \mathrm{~km}$, as well as the Sava Bohinjka source, the river flows through agricultural areas and the urban centres of Jesenice, Kranj, Ljubljana, the mining area of Zasavje and Krško (Figure 1).

Discharge regimes along its flow are controlled by precipitation and the configuration of the landscape. In the upper part of the Sava a snow - rain regime prevails and turns over in the central and lower part to a rain - snow regime (Hrvatin, 1998). Annual maxima are characteristics in spring and late summer, while minima occur in summer and winter months. In the years 1961-1990 the mean annual amount of precipitation in the River Sava watershed was $1500 \mathrm{~mm}$ (Zupančič, 1998). Long term (from the years 1960-1991) the mean annual discharge varies at the gauging stations of Radovljica (in the upper part of the watershed), Hrastnik (in the central part of the watershed) and čatež (in the lower part of the watershed) from 18.6, 181.7 and $290 \mathrm{~m}^{3} / \mathrm{s}$, respectively (ARSO, 2004). Discharge ratios between high and low waters are $1: 100$ (extreme $1: 250$ ) and are also controlled by hydroelectric power plant outflows. 


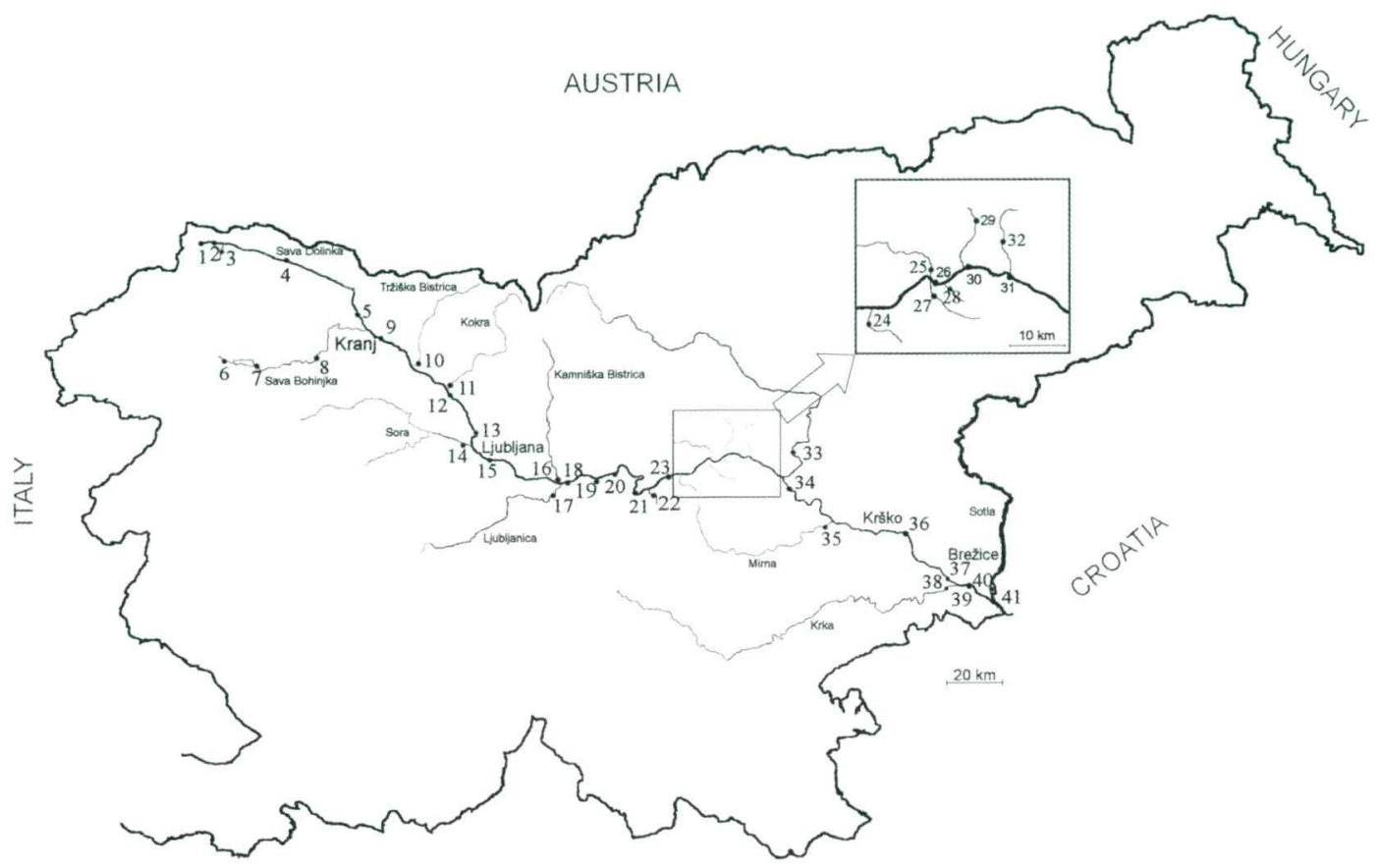

Figure 1. Detailed location map of the numbered sampling sites in the River Sava watershed. Sample sites are described in Table 1.

The drainage area of the River Sava in Slovenia is $10881 \mathrm{~km}^{2}$ (ARSO, 2004).

The valley of the Sava extends in a NWSE direction and comprises almost half the Slovenian territory with variegated geological composition. At the confluence of the Sava Bohinjka and Sava Dolinka it accumulates Pleistocene fluvioglacial sediments and formed terraces, from Ljubljana the watershed is mainly composed of Permo-Carbonian clastic sediments, which alternate with Triassic carbonates in the Zasavje area and pass over to Miocene sandstones, clays and gravels on the left bank of the river. In the Krško-Brežice area the watershed mainly consists of terrace Pleistocene sediments and from there the flow continues to Croatia.

The watershed of the Sava's tributaries is composed of Triassic and Jurassic carbonates, Permo-Carbonian, Oligocene, Miocene clastic rocks and Pleistocene sediments (Buser \& Draksler, 1989).

\section{Methodology}

Surface water sampling locations were selected based on their relationship to the confluences of major and minor streams, at points before and after the confluence. Sampling locations of the Sava watershed are presented in Figure 1. Sampling was performed at 41 locations in different seasons (April - spring 2004, September - late summer 2004 and January - winter 2005), according to the discharge regimes of the Sava and its tributaries.

Temperature, conductivity, dissolved oxygen (DO), and $\mathrm{pH}$ measurements were performed in the field. Sample aliquots collected for chemical analysis were immediately passed through a $0.45 \mu \mathrm{m}$ nylon filter into bottles and kept refrigerated until analysed. Samples for cation (pre-treated with $\mathrm{HNO}_{3}$ ), anion and alkalinity analyses were preserved in HDPE bottles. Samples for DIC (dissolved inorganic carbon) and $\delta^{13} \mathrm{C}_{\mathrm{DIC}}$ analyses were stored in glass bottles, filled to the top, with no headspace. Samples for DOC analyses were acidified and sampled in glass bottles of volume $30 \mathrm{ml}$. Samples for isotopic composition of sulphur were collected in $4.5 \mathrm{l}$ plastic bottles and were acidified after filtration to $\mathrm{pH} 2$. Then $\mathrm{BaCl}_{2}$ was added to precipitate $\mathrm{BaSO}_{4}$, which was then collected by filtering through a $0.45 \mu \mathrm{m}$ fil- 
ter. Sampling of water samples for $\delta^{18} \mathrm{O}$ and $\delta \mathrm{D}$ analyses was performed in plastic bottles of volume $50 \mathrm{ml}$ filled to the top.

Total alkalinity was measured by Gran titration with a precision of $\pm 1 \%$ within $24 \mathrm{~h}$ of sample collection. Major elements $\left(\mathrm{Ca}^{2+}, \mathrm{Mg}^{2+}, \mathrm{Na}^{+}, \mathrm{K}^{+}, \mathrm{Sr}^{2+}, \mathrm{Si}, \mathrm{NO}_{3}^{-}, \mathrm{SO}_{4}{ }^{2-}, \mathrm{Cl}^{-}\right)$ in water samples were determined by Inductively Coupled Plasma-Optical Emission Spectroscopy (ICP-OES) and ion chromatography, with a precision of $\pm 2 \%$. Dissolved organic carbon (DOC) concentrations were measured using a high-temperature platinum-catalyzed carbon analyzer via combustion, followed by infrared detection of $\mathrm{CO}_{2}$ (Shmidizu TOC-5000A) with an uncertainty of $\pm 2 \%$. The concentrations of DIC were determined on a UIC Coulometrics $\mathrm{CO}_{2}$ Coulometer with a precision of $\pm 2 \%$.

Isotopic composition of light elements $(\mathrm{H}$, $\mathrm{C}, \mathrm{N}, \mathrm{O}, \mathrm{S}$ ) in sample is expressed as $\delta$ value as relative difference of stable isotope ratios with regard to default reference material as follows:

$$
\delta_{v z}=\frac{R_{V Z}-R_{R M}}{R_{R M}} \cdot 1000 \quad[\% \mathrm{o}]
$$

Value $\mathrm{R}$ is ratio between heavier stable isotope and lighter stable isotope: $\mathrm{D} / \mathrm{H}$, ${ }^{13} \mathrm{C} /{ }^{12} \mathrm{C},{ }^{15} \mathrm{~N} /{ }^{14} \mathrm{~N},{ }^{18} \mathrm{O} /{ }^{16} \mathrm{O},{ }^{34} \mathrm{~S} /{ }^{32} \mathrm{~S}$.

Positive $\delta$ values (expressed in \%) mean that sample contains more heavier isotope than reference material, and negative that it contains less (O'Neil, 1979).

Isotopic reference materials are determined by International Atomic Energy Agency (IAEA) and National Institute of Standards and Technology (NIST). For each of the element the reference materials are selected so that isotopic ratio in reference material is similar to average ratio of the same isotopes in nature.

At measurments of isotopic composition of oxygen (hydrogen), carbon, nitrogen and sulphur the following reference materials are used:

- VSMOW - Vienna Standard Mean Ocean Water

- VPDB - Vienna Pee Dee Belemnite

- AIR - Air

- VCDT - Vienna Cañon Diablo Troilite

The stable isotope composition of dissolved inorganic carbon $\left(\delta^{13} \mathrm{C}_{\text {DIC }}\right)$ was determined with a Europa Scientific 20-20 continuous flow IRMS ANCA - TG preparation module. Phosphoric acid (100 \%) was added
$(100-200 \mu \mathrm{l})$ to a septum-sealed vial which was then purged with pure He. The water sample $(6 \mathrm{ml})$ was injected into the septum tube and headspace $\mathrm{CO}_{2}$ was measured. In order to determine the optimal extraction procedure for surface water samples, a standard solution of $\mathrm{Na}_{2} \mathrm{CO}_{3}$ (Carlo Erba) with a known $\delta^{13} \mathrm{C}_{\mathrm{DIC}}$ of $-10.8 \pm 0.2 \%$ o was prepared with a concentration of either $4.8 \mathrm{mmol} / \mathrm{l}$ (for samples with an alkalinity above $2 \mathrm{mmol} / \mathrm{l}$ ) or of $2.4 \mathrm{mmol} / \mathrm{l}$ (for samples with alkalinity below $2 \mathrm{mmol} / \mathrm{l}$ ). Since no reference material for $\delta^{13} \mathrm{C}_{\mathrm{DIC}}$ measurements exists, $\mathrm{CO}_{2}$ was used with $\delta^{13} \mathrm{C}_{\mathrm{CO} 2}-5.2$ $\pm 0.2 \%$ as a laboratory standard, which is calibrated to international reference materials: NIST 8562, NIST 8563 and NIST 8564 with default $\delta$ value relative to VPDB.

The isotopic composition of oxygen and hydrogen in water $\left(\delta^{18} \mathrm{O}\right.$ and $\left.\delta \mathrm{D}\right)$ were measured with a Varian Mat 250. The same reference materials (NIST) were used for $\delta^{18} \mathrm{O}$ and for $\delta^{13} \mathrm{C}_{\mathrm{DIC}}$ measurements as described above. For measurement control of $\delta \mathrm{D}$ value IAEA OH4 and IAEA OH1 reference materials were used with $-109 \% \pm 1.5$ and $-3.9 \%$ \pm 1.5 to relate analytical results to VSMOW.

The stable isotope composition of sulphur $\left(\delta^{34} \mathrm{~S}_{\mathrm{SO} 4}\right)$ was determined with a Europa Scientific 20-20 continuous flow IRMS ANCA - SL preparation module. About $10 \mathrm{mg}$ of $\mathrm{BaSO}_{4}$ was scraped from the filters and transferred to a tin capsule. $\delta^{34} \mathrm{~S}_{\mathrm{SO} 4}$ was determined after combustion $\left(1000{ }^{\circ} \mathrm{C}\right)$ of the capsule and reduction in a $\mathrm{Cu}$ tube $\left(600^{\circ} \mathrm{C}\right)$. NBS 127 and NBS 22 reference materials for sulphur were used to relate analytical results to the VCDT standard.

Partial pressure of $\mathrm{CO}_{2}\left(\mathrm{p}_{\mathrm{CO} 2}\right)$, as well as saturation indexes of calcite and dolomite $\left(\mathrm{SI}_{\text {calcite }}, \mathrm{SI}_{\text {dolomite }}\right)$, were calculated by the PHREEQC for Windows program (P arkhurst \& Appelo, 1999).

\section{Results and discussion \\ Major chemistry}

The concentration of $\mathrm{Ca}^{2+}$ in Sava water seasonally changes from 39.9 to $60.8 \mathrm{mg} / \mathrm{l}$ in spring 2004, from 36.5 to $62.8 \mathrm{mg} / \mathrm{l}$ in late summer and from 39.2 to $72.8 \mathrm{mg} / \mathrm{l}$ in winter 2005. The concentration of $\mathrm{Ca}^{2+}$ in water of Sava tributaries seasonally ranges from 5.95 to $93.5 \mathrm{mg} / \mathrm{l}$ in spring 2004 , from 11.9 to 95.4 $\mathrm{mg} / \mathrm{l}$ in late summer 2004 and from 6.76 to $96.2 \mathrm{mg} / \mathrm{l}$ in winter 2005 , respectively ( $\mathrm{Ta}-$ bles 1, 2 and 3 ). 
The concentration of $\mathrm{Mg}^{2+}$ in Sava water changes from 8.0 to $12.8 \mathrm{mg} / \mathrm{l}$ in spring 2004 , from 10.1 to $13.7 \mathrm{mg} / \mathrm{l}$ in late summer and from 11.8 to $16.7 \mathrm{mg} / \mathrm{l}$ in winter. The concentration of $\mathrm{Mg}^{2+}$ in water of Sava tributaries seasonally ranges from 1.84 to $22 \mathrm{mg} / \mathrm{l}$ in spring 2004, from 3.4 to $28 \mathrm{mg} / \mathrm{l}$ in late summer 2004 and from 1.9 to $29.9 \mathrm{mg} / \mathrm{l}$ in winter (Tables 1, 2 and 3 ).

$\mathrm{A} \mathrm{Ca}^{2+} / \mathrm{Mg}^{2+}$ molar ratio around 2 is characteristic of carbonate weathering (Meybeck, 1996) and therefore for the carbonate part of the Sava watershed (sampling locations 1-5, upper part of the watershed). Deviation from this ratio arises from additional mineralization of water due to erosion of mechanically less resistant clastics rocks in the central and lower part of the River Sava flow, where the ratios are higher and also concentrations of $\mathrm{Ca}^{2+}$ increase in comparison to the Sava Bohinjka and Sava Dolinka, where weathering of carbonates prevails (Figure 2). The $\mathrm{Ca}^{2+} / \mathrm{Mg}^{2+}$ ratio also increases at the confluence of the Sava Dolinka and Bohinjka (9) in the spring sampling season due to snow melting and higher amounts of precipitation. The $\mathrm{Ca}^{2+} / \mathrm{Mg}^{2+}$ ratio also indicates the relative contributions of calcite and dolomite which contribute to the chemical composition of water. Dolomite weathering give a $\mathrm{Ca}^{2+} / \mathrm{Mg}^{2+}$ molar ratio around 1 , while carbonate weathering gives a ratio around 2 (Pawellek et al., 2002). In the case of weathering of calcite as the dominant mineral, the $\mathrm{Ca}^{2+} / \mathrm{Mg}^{2+}$ ratio rises above 5. From the $\mathrm{Ca}^{2+} / \mathrm{Mg}^{2+}$ ratio in the Sava watershed it can be concluded that its chemical composition is mainly controlled by weathering of calcite with magnesium. Precipitation of tufa is a common process ( $R$ a mov $\breve{s}, 1983$ ) at location 28 in the late summer season (low discharge conditions), which is reflected in the $\mathrm{Ca}^{2+} / \mathrm{Mg}^{2+}$ ratio. In the late summer sampling season the $\mathrm{Ca}^{2+} / \mathrm{Mg}^{2+}$ ratio is 2.7 , while in spring it increases to 4.5 .

Saturation indexes for the Sava watershed were calculated by the PHREEQC program for Windows (Parkhurst \& Appelo, 1999). Thermodynamically calculated of saturation indexes of carbonates $\left(\mathrm{SI}_{\text {calcite: }}\right.$ $\mathrm{SI}_{\text {dolomite }}$ ) were in the range of 0.5 froin equilibrium (Appelo \& Postma, 1994) and are presented for the River Sava system in Tables 1,2 and 3 . These saturation indexes indicate precipitation of carbonate in the central and lower Sava River flow, while in the upper flow the index rarely reaches satura- tion. The stable isotope composition of particulate inorganic carbon $\left(\delta^{3} \mathrm{C}_{\mathrm{PIC}}\right)$ measured from the River Sava watershed varied from 0.5 to $2.1 \%$ with an average of $1.3 \pm 0.5 \%$ $(n=9)$, virtually identical in value that of carbonate rocks (Kanduč, 2006). This suggests that suspended carbonate material in these streams is detrital in origin and does not represent authigenic carbonate formed in response to the high degrees of supersaturation with respect to calcite.

The partial pressure of $\mathrm{CO}_{2}$ in the Sava watershed changed from $158.5 \mathrm{ppm}$ to 2951 ppm in spring 2004, from 446 to $9120 \mathrm{ppm}$ in late summer and from 549 to $2290 \mathrm{ppm}$ in winter (Tables 1, 2 and 3). The highest partial pressure was observed in the late summer season probably due to degradation of organic matter in soils leaching into the river and/or in the river itself (Dever et al., 1983). The higher partial pressure in the winter sampling season could also be attributed to higher $\mathrm{CO}_{2}$ dissolution in water in the winter months (Atkins, 1994). The River Sava in Slovenia shows seasonal patterns of $\mathrm{CO}_{2}$ flux between the water - atmosphere interface similar to other large rivers, like the Rhine (3300 ppm), Amazon (4000 ppm) and Yangtze (from 3160 to $4700 \mathrm{ppm}$ ) (Gao \& Kempe, 1987).

Alkalinity in the River Sava ranged from 2.61 to $3.75 \mathrm{mmol} / \mathrm{l}$ in spring 2004, from 2.63 to $4.76 \mathrm{mmol} / \mathrm{l}$ in late summer 2004 and from 2.67 to $4.22 \mathrm{mmol} / \mathrm{l}$ in winter 2005 , respectively. Alkalinity ranged from 0.38 to 5.22 $\mathrm{mmol} / \mathrm{l}$ in spring 2004, from 0.84 to 6.02 $\mathrm{mmol} / \mathrm{l}$ in late summer 2004 and from 0.44 to $6.30 \mathrm{mmol} / \mathrm{l}$ in winter 2005 (Tables 1, 2 and 3 ). This seasonal variability of alkalinity is due to discharge conditions as well as processes in the River Sava watershed (Figures $3 \mathrm{~A}$ and $3 \mathrm{~B}$ ). Lower alkalinities are observed in the spring sampling season due to dilution of river water by precipitation with an alkalinity around $0 \mathrm{mmol} / \mathrm{l}$, while in late summer the higher alkalinity could be attributed to oxic degradation of organic matter. In winter 2005 discharges, degradation processes and leaching from the terrestrial to the river ecosystem are limited and consequently alkalinities are higher in comparison with the spring sampling season. In late summer higher alkalinities are observed due to lower discharge conditions and higher degradation of organic matter. Alkalinity is also related to the geological composition of the River Sava watershed. The upper part 
Table 1. Chemical and isotopic data for the River Sava watershed, spring 2004. Locations are plotted on Figure 1.

\begin{tabular}{|c|c|c|c|c|c|c|c|c|c|c|c|}
\hline $\begin{array}{l}\text { Sampling } \\
\text { point }\end{array}$ & $Q\left(\mathrm{~m}^{3} / \mathrm{s}\right)$ & $\begin{array}{c}\mathrm{T} \\
\left({ }^{\circ} \mathrm{C}\right)\end{array}$ & $\begin{array}{l}\text { D. } 0 . \\
(\%)\end{array}$ & $\begin{array}{c}\text { Conductivity } \\
(\mu \mathrm{S} / \mathrm{cm})\end{array}$ & $\mathrm{pH}$ & $\begin{array}{c}\text { Alkalinity } \\
(\mathrm{mmol} / \mathrm{l})\end{array}$ & $\begin{array}{c}\text { DIC } \\
(\mathrm{mmol} / \mathrm{I})\end{array}$ & $\begin{array}{l}\text { DOC } \\
(\mathrm{mg} / \mathrm{l})\end{array}$ & $\begin{array}{c}\mathrm{Ca} \\
(\mathrm{mg} / \mathrm{l})\end{array}$ & $\begin{array}{c}\mathrm{Mg} \\
(\mathrm{mg} / \mathrm{l})\end{array}$ & $\begin{array}{c}\mathrm{Na} \\
(\mathrm{mg} / \mathrm{l})\end{array}$ \\
\hline 1 & & 8.6 & 88.0 & 276 & 7.83 & 2.98 & 2.98 & 3.83 & 40.75 & 12.60 & 1.23 \\
\hline 2 & 1.55 & 8.9 & 96.0 & 244 & 8.14 & 2.62 & 2.54 & 2.64 & & & \\
\hline 3 & & 7.6 & 94.5 & 216 & 8.36 & 2.37 & 2.29 & 3.11 & 32.94 & 10.64 & 1.59 \\
\hline 4 & 11.4 & 9.7 & 99.3 & 251 & 8.28 & 2.81 & 2.69 & 4.07 & 39.96 & 12.81 & 1.60 \\
\hline 5 & 28.6 & 9.7 & 98.6 & 283 & 8.48 & 3.19 & 2.81 & 2.76 & 45.09 & 10.40 & 2.18 \\
\hline 6 & n.a. & 6.2 & 102.5 & 172 & 8.32 & 2.00 & 1.65 & 3.03 & 30.32 & 4.39 & 0.16 \\
\hline 7 & 3.75 & 12.3 & 112.7 & 201 & 8.26 & 2.35 & 2.07 & 2.66 & 36.01 & 4.88 & 1.11 \\
\hline 8 & 10.5 & 9.5 & 96.0 & 203 & 8.43 & 2.30 & 2.24 & 1.48 & 40.50 & 4.73 & 0.66 \\
\hline 9 & 39.9 & 10.3 & 102.6 & 254 & 8.57 & 2.91 & 2.47 & 2.23 & 44.04 & 8.00 & 1.60 \\
\hline 10 & 2.37 & 11.0 & 111.4 & 277 & 8.95 & 2.60 & & 2.27 & 48.73 & 10.74 & 1.99 \\
\hline 11 & 42.1 & 12.1 & 100.5 & 264 & 8.10 & 2.64 & 2.45 & 1.51 & 43.20 & 10.52 & 1.71 \\
\hline 12 & 31.9 & 10.5 & 108.8 & 246 & 8.59 & 2.89 & 2.60 & 3.45 & 45.93 & 8.53 & 1.77 \\
\hline 13 & 67.2 & 10.6 & 86.5 & 305 & 8.42 & 2.79 & 2.73 & 3.11 & 47.38 & 9.19 & 2.93 \\
\hline 14 & 4.32 & 10.2 & 94.7 & 260 & 8.37 & 2.48 & 2.43 & 1.18 & 37.48 & 10.45 & 2.75 \\
\hline 15 & & 10.4 & 102.8 & 302 & 8.43 & 3.16 & 2.77 & 1.67 & 46.21 & 9.49 & 2.23 \\
\hline 16 & 0.731 & 11.2 & 107.0 & 381 & 8.17 & 3.90 & & 2.09 & 56.88 & 11.26 & 8.09 \\
\hline 17 & 7.22 & 10.9 & 97.0 & 391 & 8.10 & 4.01 & 3.65 & 2.36 & 58.89 & 12.21 & 3.90 \\
\hline 18 & & 10.9 & 110.2 & 338 & 8.23 & 3.27 & 3.15 & 1.45 & 54.42 & 10.94 & 3.97 \\
\hline 19 & & 13.0 & 99.4 & 62 & 7.79 & 0.39 & 0.44 & 3.88 & 5.95 & 1.84 & 2.99 \\
\hline 20 & & 11.1 & 105.5 & 324 & 8.20 & 3.60 & 3.15 & 1.58 & 53.16 & 10.92 & 3.85 \\
\hline 21 & 105 & 12.1 & 114.0 & 323 & 8.27 & 3.28 & & 2.41 & 53.58 & 11.04 & 3.74 \\
\hline 22 & & 13.4 & 99.0 & 272 & 8.18 & 2.59 & 2.51 & & 32.12 & 15.09 & 4.02 \\
\hline 23 & & 11.2 & 90.0 & 344 & 8.18 & 3.00 & 2.94 & 1.63 & 50.44 & 10.96 & 3.52 \\
\hline 24 & & 8.4 & 93.1 & 208 & 8.17 & 1.87 & & & 22.82 & 10.85 & 3.43 \\
\hline 25 & 1 & 8.7 & 91.0 & 491 & 8.16 & 4.15 & 3.98 & & 65.88 & 18.52 & 6.69 \\
\hline 26 & & 10.4 & 87.4 & 380 & 8.10 & 3.32 & 3.27 & & 53.78 & 11.59 & 3.96 \\
\hline 27 & & 8.7 & 98.0 & 424 & 8.34 & 4.26 & 4.15 & & 59.17 & 22.01 & 3.35 \\
\hline 28 & & 8.4 & 95.0 & 318 & 8.19 & 3.34 & 3.32 & & 63.14 & 8.26 & 0.74 \\
\hline 29 & & 10.1 & 89.2 & 389 & 8.31 & 3.56 & 2.69 & & 36.39 & 18.36 & 8.89 \\
\hline 30 & & 10.7 & 84.2 & 395 & 8.08 & 3.57 & 3.27 & & 54.98 & 11.95 & 4.49 \\
\hline 31 & 92.2 & 10.8 & 84.6 & 349 & 8.08 & 3.38 & 2.95 & & 53.03 & 11.31 & 4.04 \\
\hline 32 & & 11.0 & 93.7 & 573 & 8.10 & 4.48 & 4.10 & & & & \\
\hline 33 & 25 & 14.4 & 127.8 & 375 & 8.98 & 3.04 & 2.74 & 6.53 & 52.07 & 10.56 & 7.40 \\
\hline 34 & & 11.6 & 66.7 & 375 & 8.10 & 3.66 & 3.27 & & 53.84 & 11.38 & 4.66 \\
\hline 35 & 1.94 & 12.9 & 95.6 & 443 & 8.48 & 4.57 & 4.27 & & 66.37 & 20.03 & 2.48 \\
\hline 36 & & 13.4 & 94.0 & 383 & 8.42 & 3.17 & 3.17 & 2.44 & 55.46 & 11.73 & 4.37 \\
\hline 37 & & 14.2 & 90.2 & 396 & 8.04 & 3.39 & 3.25 & & 56.82 & 12.63 & 4.86 \\
\hline 38 & 26.9 & 13.5 & 105.5 & 423 & 8.32 & 4.33 & 3.75 & & 66.81 & 12.24 & 2.55 \\
\hline 39 & 120 & 13.4 & 98.0 & 412 & 8.28 & 3.52 & 3.53 & & 60.81 & 12.24 & 3.60 \\
\hline 40 & 1.8 & 14.0 & 91.5 & 570 & 8.15 & 5.22 & 5.09 & 3.85 & 93.46 & 16.61 & 8.56 \\
\hline 41 & 144 & 13.8 & 106.3 & 398 & 8.23 & 3.75 & 3.47 & & 59.39 & 12.26 & 4.02 \\
\hline
\end{tabular}

of the watershed is composed of carbonate rocks, where low alkalinities are observed, while in the central and lower flow higher alkalinities are observed since the watershed is composed of carbonate and clastic rocks.

The annual flow of dissolved inorganic carbon $\left(\mathrm{F}_{\text {DIC }}\right)$, calculated from carbonate alkalinity and discharges (EIONET, 2005) was estimated to be $4.1 \times 10^{11} \mathrm{~g} \mathrm{C} /$ year or $4.0 \times$ $10^{7} \mathrm{~g} \mathrm{C} /\left(\right.$ year $\mathrm{km}^{2}$ ) at the Bregana (41) location, according to the drainage area of the River Sava in Slovenia.

In Tables 1, 2 and 3 results of other cation $\left(\mathrm{Na}^{+}, \mathrm{K}^{+}, \mathrm{Sr}^{2+}\right.$ and $\left.\mathrm{Si}\right)$ and anion $\left(\mathrm{NO}_{3}{ }^{-}, \mathrm{SO}_{4}{ }^{2-}\right.$ and $\left.\mathrm{Cl}^{-}\right)$analyses are also presented. 


\begin{tabular}{|c|c|c|c|c|c|c|c|c|c|c|c|c|c|}
\hline $\begin{array}{c}\mathrm{K} \\
(\mathrm{mg} / \mathrm{l})\end{array}$ & $\begin{array}{c}\mathrm{Sr} \\
(\mathrm{mg} / \mathrm{l})\end{array}$ & $\begin{array}{c}\mathrm{Si} \\
(\mathrm{mg} / \mathrm{l})\end{array}$ & $\begin{array}{c}\mathrm{NO}_{3} \\
(\mathrm{mg} / \mathrm{l})\end{array}$ & $\begin{array}{c}\mathrm{SO}_{4} \\
(\mathrm{mg} / \mathrm{l}) \\
\end{array}$ & $\begin{array}{c}\mathrm{Cl} \\
(\mathrm{mg} / \mathrm{l})\end{array}$ & $\mathrm{SI}_{\text {calcite }}$ & $\mathrm{SI}_{\text {dolomite }}$ & $\begin{array}{c}\mathrm{p}_{\mathrm{cO} 2} \\
(\mathrm{ppm})\end{array}$ & $\begin{array}{c}\delta^{13} \mathrm{C}_{\mathrm{DIC}} \\
(\% \mathrm{o})\end{array}$ & $\begin{array}{l}\delta^{18} O \\
(\% 0)\end{array}$ & $\begin{array}{l}\delta D \\
(\%)\end{array}$ & d $(\% 0)$ & $\begin{array}{c}\delta^{34} \mathbf{S}_{\mathrm{SO} 4} \\
(\% 0)\end{array}$ \\
\hline \multirow[t]{2}{*}{0.00} & \multirow[t]{2}{*}{0.02} & \multirow[t]{2}{*}{0.72} & 2.11 & 6.01 & 0.04 & \multirow[t]{2}{*}{0.04} & \multirow[t]{2}{*}{-0.34} & 2138.0 & -10.7 & -10.4 & -65.5 & 16.66 & \\
\hline & & & 1.39 & 9.24 & 5.27 & & & 158.5 & -10.3 & -10.2 & -64.1 & 16.48 & \\
\hline 0.00 & 0.10 & 0.35 & 2.24 & 6.39 & 0.04 & 0.36 & 0.31 & 489.8 & -7.9 & -10.5 & -67.1 & 15.85 & \\
\hline 0.00 & 0.15 & 0.72 & 2.05 & 12.29 & 0.05 & 0.45 & 0.54 & 707.9 & -8.6 & -10.5 & -67.9 & 15.05 & 9.5 \\
\hline 0.00 & 0.12 & 0.70 & 2.53 & 14.57 & 0.16 & 0.75 & 0.98 & 501.2 & -9.6 & -11.0 & -65.5 & 21.4 & \\
\hline 0.00 & 0.01 & 0.14 & 2.55 & 2.86 & 0.02 & 0.21 & -0.37 & 457.1 & -5.8 & -12.4 & -80.0 & 17.96 & \\
\hline 0.00 & 0.01 & 0.30 & 2.55 & 3.76 & 0.02 & 0.38 & 0.06 & 645.7 & -8.3 & -10.2 & -62.8 & 17.78 & \\
\hline 0.00 & 0.02 & 0.37 & 2.64 & 4.02 & 1.17 & 0.54 & 0.26 & 407.4 & -9.1 & -10.6 & -71.5 & 12.24 & \\
\hline 0.00 & 0.07 & 0.55 & 2.76 & 8.78 & 0.09 & 0.8 & 0.99 & 371.5 & -9.0 & -10.8 & -66.0 & 19.32 & \\
\hline 0.00 & 0.32 & 0.81 & 2.46 & 34.95 & 0.13 & 1.12 & 1.73 & 128.8 & -6.8 & -10.5 & -66.0 & 16.95 & 14.5 \\
\hline 0.00 & 0.12 & 0.45 & 2.62 & 18.02 & 0.05 & 0.33 & 0.2 & 1047.1 & -7.0 & -10.3 & -66.7 & 14.67 & 9.4 \\
\hline 0.24 & 0.09 & 0.62 & 2.94 & 11.97 & 2.97 & 0.83 & 1.07 & 354.8 & -9.5 & -10.6 & -66.4 & 17.34 & 10.4 \\
\hline 1.12 & 0.09 & 0.68 & 3.95 & 12.98 & 0.08 & 0.67 & 0.76 & 512.9 & -10.2 & -10.2 & -69.7 & 10.88 & 9.6 \\
\hline 0.35 & 0.07 & 1.31 & 5.32 & 11.15 & 0.05 & 0.48 & 0.52 & 512.9 & -11.2 & -9.3 & -61.5 & 11.97 & 3.9 \\
\hline 0.05 & 0.09 & 0.72 & 4.62 & 12.97 & 0.07 & 0.72 & 0.88 & 562.3 & -10.7 & -10.1 & -67.0 & 12.79 & \\
\hline 1.30 & 0.06 & 1.03 & 13.00 & 16.46 & 0.07 & 0.64 & 0.72 & 1288.2 & -12.4 & -9.2 & -60.9 & 11.78 & 1.8 \\
\hline 0.13 & 0.06 & 0.70 & & & & 0.6 & 0.67 & 1548.8 & -13.5 & -10.0 & -66.3 & 12.7 & 7.8 \\
\hline 0.15 & 0.08 & 0.78 & 6.13 & 14.15 & 0.06 & 0.61 & 0.66 & 933.3 & -12.7 & -9.9 & -68.0 & 10.21 & \\
\hline 1.14 & 0.03 & 4.45 & 1.84 & 6.35 & 2.65 & -1.56 & -3.46 & 338.8 & -8.1 & -9.1 & -57.6 & 14.29 & \\
\hline 0.47 & 0.07 & 0.78 & 1.84 & 13.73 & 0.06 & 0.6 & 0.68 & 1096.5 & -12.5 & -9.8 & & & \\
\hline 0.14 & 0.08 & 0.74 & 1.84 & 14.22 & 0.06 & 0.66 & 0.79 & 871.0 & -12.3 & -9.8 & & & \\
\hline 1.23 & 0.03 & 3.03 & 1.84 & 13.36 & 0.06 & 0.29 & 0.43 & 891.3 & -13.2 & -9.4 & & & \\
\hline 0.24 & 0.08 & 0.82 & 1.84 & 13.69 & 5.72 & 0.5 & 0.48 & 977.2 & -12.0 & -9.7 & -63.3 & 13.33 & 7.1 \\
\hline 1.26 & 0.05 & 3.99 & 1.84 & 14.51 & 3.33 & -0.06 & -0.36 & 616.6 & -11.6 & -9.6 & -61.2 & 14.64 & \\
\hline 2.11 & 0.14 & 2.76 & 1.84 & 36.24 & 0.13 & 0.66 & 0.87 & 1349.0 & -13.0 & -9.8 & -61.0 & 16.42 & \\
\hline 0.19 & 0.08 & 0.72 & 1.84 & 15.43 & 5.85 & 0.48 & 0.41 & 1288.2 & -11.0 & -10.4 & -68.0 & 14.16 & 6.1 \\
\hline 0.48 & 0.06 & 1.49 & 1.84 & 24.26 & 5.77 & 0.8 & 1.28 & 912.0 & -12.4 & -10.2 & -63.4 & 17.18 & \\
\hline 0.09 & 0.02 & 0.88 & 1.84 & 16.13 & 1.74 & & 0.41 & 1023.3 & -12.6 & -10.2 & & & 8.5 \\
\hline 1.73 & 0.07 & 3.34 & 1.84 & 26.85 & 0.18 & 0.53 & 0.89 & 831.8 & -10.5 & -10.3 & -72.3 & 9.07 & 5.5 \\
\hline 0.53 & 0.09 & 0.76 & 1.84 & 19.79 & 6.22 & 0.5 & 0.46 & 1445.4 & -11.5 & -10.0 & & & 4.2 \\
\hline 0.32 & 0.08 & 0.66 & 1.84 & 15.38 & 6.19 & 0.46 & 0.39 & 1380.4 & -11.5 & -10.7 & -70.5 & 14.03 & 8.5 \\
\hline & & & 1.84 & 108.37 & 7.50 & & & 1819.7 & -9.5 & -10.1 & -62.6 & 17.19 & \\
\hline 1.13 & 0.15 & 0.89 & 1.84 & 28.35 & 0.08 & 1.27 & 2.06 & 141.3 & -8.5 & -10.2 & -67.0 & 13.58 & 4.3 \\
\hline 0.74 & 0.10 & 0.74 & 1.84 & 18.61 & 0.08 & 0.53 & 0.54 & 1445.4 & -11.1 & -10.2 & -64.7 & 15.88 & 3.7 \\
\hline 0.14 & 0.11 & 1.87 & 1.8 & 17.97 & 0.08 & 1.07 & 1.8 & 724.4 & -11.5 & -10.0 & -65.0 & 14 & 6.3 \\
\hline 0.44 & 0.09 & 0.74 & 1.84 & 17.05 & 5.7 & 0.82 & 1.14 & 588.8 & -10.0 & -10.6 & -65.4 & 18.34 & 3.9 \\
\hline 0.72 & 0.10 & 0.90 & 1.84 & 21.43 & & & 0.5 & 2951.2 & -11.9 & -9.7 & -55.7 & 20.93 & \\
\hline 0.11 & 0.05 & 1.02 & 1.84 & 9.81 & 0.05 & 0.92 & & 1023.3 & -12.9 & -10.1 & -69.9 & 9.89 & \\
\hline 0.16 & 0.07 & 0.92 & 1.84 & 15.12 & 5.62 & 0.76 & 1.02 & 912.0 & -11.1 & -10.5 & -64.8 & 18.15 & 3.8 \\
\hline 2.00 & 0.39 & 2.43 & 1.84 & 39.56 & 10.40 & 0.95 & 1.35 & 1819.7 & -11.8 & -10.3 & -66.5 & 14.87 & -1.3 \\
\hline 0.49 & 0.08 & 0.90 & 1.84 & 18.33 & 0.07 & 0.74 & 0.98 & 1096.5 & -11.1 & -10.2 & -68.8 & 11.78 & 5.4 \\
\hline
\end{tabular}

\section{Carbon Isotopes}

Values of $\delta^{13} \mathrm{C}_{\mathrm{DIC}}$ in the River Sava ranged from -12.7 to $-8.6 \%$ in spring 2004 , from - 11.8 to $-7.3 \%$ in late summer 2004 and from -10.6 to $-6.3 \%$ in winter 2006 (Tables 1, 2 and 3, Figure $3 \mathrm{C}$ ). In the Sava tributaries $\delta^{13} \mathrm{C}_{\text {DIC }}$ values ranged from -13.5 to -5.8 \%o in spring 2004 , from -12.8 to $-3.3 \%$ in late summer 2004 and from -11.9 to -4.2 $\%$ in winter 2005 (Tables 1, 2 and 3, Figure 3 D). Lower $\delta^{13} \mathrm{C}_{\text {DIC }}$ values in the Sava and its tributaries were observed in the spring season due to more abundant precipitation. More positive $\delta^{13} \mathrm{C}_{\text {DIC }}$ values were observed in all sampling seasons in the carbonate 
Table 2. Chemical and isotopic data for the River Sava watershed, late summer 2004. Locations are plotted on Figure 1.

\begin{tabular}{|c|c|c|c|c|c|c|c|c|c|c|}
\hline $\begin{array}{l}\text { Sampling } \\
\text { point }\end{array}$ & $\begin{array}{c}Q \\
\left(\mathrm{~m}^{3} / \mathrm{s}\right)\end{array}$ & $\begin{array}{c}\mathrm{T} \\
\left({ }^{\circ} \mathrm{C}\right)\end{array}$ & $\begin{array}{l}\text { D.O. } \\
(\%)\end{array}$ & $\begin{array}{l}\text { Conductivity } \\
(\mu \mathrm{S} / \mathrm{cm})\end{array}$ & $\mathrm{pH}$ & $\begin{array}{c}\text { Alkalinity } \\
(\mathrm{mmo} / \mathrm{l})\end{array}$ & $\begin{array}{c}\mathrm{DOC} \\
(\mathrm{mg} / \mathrm{l})\end{array}$ & $\begin{array}{c}\mathrm{Ca} \\
(\mathrm{mg} / \mathrm{l})\end{array}$ & $\begin{array}{c}\mathrm{Mg} \\
(\mathrm{mg} / \mathrm{l})\end{array}$ & $\begin{array}{l}\mathrm{Na} \\
(\mathrm{mg} / \mathrm{l})\end{array}$ \\
\hline 1 & & 5.6 & 110.0 & 279 & 7.54 & 2.63 & 9.17 & 36.57 & 11.40 & 1.55 \\
\hline 2 & 1.55 & 7.1 & 103.0 & 380 & 7.56 & 2.67 & 5.94 & 37.45 & 11.51 & 2.95 \\
\hline 3 & & 7.6 & 114.0 & 239 & 7.82 & 3.36 & 5.50 & 34.36 & 10.75 & 1.04 \\
\hline 4 & 11.4 & 8.5 & 110.0 & 286 & 7.86 & 2.65 & 7.13 & 40.04 & 12.32 & 1.62 \\
\hline 5 & 28.6 & 10.7 & 118.0 & 311 & 8.31 & 3.06 & 10.75 & 44.88 & 12.28 & 2.35 \\
\hline 6 & n.a. & 6.0 & 116.0 & 190 & 8.08 & 2.19 & 13.72 & 30.14 & 5.49 & 0.21 \\
\hline 7 & 3.75 & 9.5 & 104.0 & 369 & 7.85 & 4.11 & 7.17 & 93.62 & 12.41 & 6.39 \\
\hline 8 & 10.5 & 12.0 & 118.0 & 247 & 8.11 & 2.69 & 5.23 & 43.15 & 5.80 & 0.83 \\
\hline 9 & 39.9 & 12.2 & 112.0 & 305 & 8.26 & 3.24 & 6.99 & 47.22 & 11.33 & 2.46 \\
\hline 10 & 2.37 & 11.3 & 107.0 & 378 & 8.44 & 2.81 & 4.89 & 55.22 & 14.50 & 2.43 \\
\hline 11 & 42.1 & 10.7 & 109.0 & 333 & 8.20 & 3.22 & 9.71 & 49.24 & 13.05 & 2.15 \\
\hline 12 & 31.9 & 14.7 & 107.0 & 632 & 7.57 & 3.22 & 6.57 & 55.21 & 12.55 & 3.88 \\
\hline 13 & 67.2 & 12.8 & 109.0 & 307 & 8.17 & 4.76 & 9.66 & 48.82 & 10.14 & 2.61 \\
\hline 14 & 4.32 & 12.5 & 104.0 & 379 & 7.48 & 4.51 & 5.48 & 55.20 & 15.39 & 4.45 \\
\hline 15 & & 13.2 & 102.0 & 379 & 7.32 & 2.99 & 6.26 & & & \\
\hline 16 & 0.731 & 13.8 & 99.0 & 554 & 7.74 & 4.6 & 6.35 & 68.81 & 14.19 & 16.95 \\
\hline 17 & 7.22 & 15.7 & 96.0 & 500 & 7.93 & 4.79 & 13.85 & 68.95 & 18.03 & 11.87 \\
\hline 18 & & 14.0 & 106.0 & 366 & 8.08 & 3.44 & 6.92 & 53.78 & 12.04 & 5.20 \\
\hline 19 & & 11.9 & 100.0 & 118.5 & 7.24 & 0.84 & & 11.98 & 3.39 & 3.72 \\
\hline 20 & & 11.5 & 100.0 & 387 & 7.29 & 3.58 & 5.99 & 58.14 & 12.01 & 4.03 \\
\hline 21 & 105 & 12.1 & 100.0 & 393 & 7.81 & 3.48 & 6.42 & 59.23 & 12.03 & 5.22 \\
\hline 22 & & 13.6 & 96.0 & 416 & 7.89 & 3.93 & 6.53 & & & \\
\hline 23 & & 13.6 & 106.0 & 378 & 8.32 & 3.81 & 6.53 & 57.56 & 11.84 & 3.99 \\
\hline 24 & & 12.7 & 108.0 & 316 & 8.34 & 2.59 & 12.38 & & & \\
\hline 25 & 1 & 18.5 & 99.0 & 574 & 8.64 & 4.89 & 3.58 & 67.73 & 24.30 & 9.35 \\
\hline 26 & & 13.7 & 95.0 & 403 & 8.35 & 3.63 & & 59.77 & 12.53 & 4.10 \\
\hline 27 & & 14.7 & 85.0 & 487 & 8.74 & 5.15 & 5.11 & 60.90 & & 2.72 \\
\hline 28 & & 15.0 & 95.0 & 414 & 8.72 & 4.03 & 3.01 & 61.80 & 13.44 & 1.44 \\
\hline 29 & & 12.5 & 94.0 & 512 & 8.04 & 4.22 & 18.66 & 56.42 & 24.22 & 16.40 \\
\hline 30 & & 17.2 & 96.0 & 403 & 8.48 & 3.54 & 4.09 & 59.39 & 12.73 & 4.95 \\
\hline 31 & 92.2 & 13.5 & 100.0 & 376 & 8.52 & 3.45 & 3.20 & 57.34 & 12.34 & 4.48 \\
\hline 32 & & 17.3 & 64.0 & 575 & 8.34 & 3.42 & 11.10 & 66.85 & 24.65 & 17.68 \\
\hline 33 & 25 & 14.3 & & 473 & 8.82 & 3.42 & & 64.97 & 13.70 & 15.71 \\
\hline 34 & & 14.4 & & 397 & 8.54 & 3.36 & 3.61 & 58.39 & 12.94 & 7.36 \\
\hline 35 & 1.94 & 14.4 & & 511 & 8.99 & 5.49 & 2.95 & 69.46 & 28.03 & 5.31 \\
\hline 36 & & 14.6 & & 453 & 8.63 & 3.6 & 5.00 & & & \\
\hline 37 & & 14.4 & & 428 & 7.86 & 3.29 & 4.86 & 62.83 & 13.67 & 8.46 \\
\hline 38 & 26.9 & 14.5 & & 459 & 8.77 & 4.65 & 3.47 & & & \\
\hline 39 & 120 & 14.7 & & 412 & 8.20 & 3.74 & 4.50 & 59.63 & 13.07 & 7.91 \\
\hline 40 & 1.8 & 12.4 & & 630 & 8.61 & 6.02 & 7.67 & 95.43 & 20.29 & 15.36 \\
\hline 41 & 144 & 14.0 & 88.0 & 424 & 7.93 & 3.51 & 6.18 & 61.15 & 13.46 & 6.89 \\
\hline
\end{tabular}

part of the watershed, while in the central and lower Sava flow, which is composed of carbonate and clastic rocks, more negative $\delta^{13} \mathrm{C}_{\mathrm{DIC}}$ values were observed. In the carbonate part of the Sava watershed lower alkalinities and lower saturation indexes were determined in comparison with the central and lower flow (Tables 1, 2 and 3). Also a negative trend of $\delta^{13} \mathrm{C}_{\mathrm{DIC}}$ values versus alkalinity was observed in all sampling seasons (Kanduč, 2006).

The isotopic composition of the source material should be studied before making an interpretation of the origin of DIC in a river ecosystem. Plants represent the source of organic material in rivers, while carbo- 


\begin{tabular}{|c|c|c|c|c|c|c|c|c|c|c|c|c|}
\hline $\begin{array}{c}\mathrm{K} \\
(\mathrm{mg} / \mathrm{l})\end{array}$ & $\begin{array}{c}\mathrm{Sr} \\
(\mathrm{mg} / \mathrm{l})\end{array}$ & $\begin{array}{c}\mathrm{Si} \\
(\mathrm{mg} / \mathrm{l})\end{array}$ & $\begin{array}{c}\mathrm{NO}_{3}{ }^{-} \\
(\mathrm{mg} / \mathrm{l})\end{array}$ & $\begin{array}{c}\mathrm{SO}_{4} \\
(\mathrm{mg} / \mathrm{l})\end{array}$ & $\begin{array}{c}\mathrm{Cl} \\
(\mathrm{mg} / \mathrm{l})\end{array}$ & $\mathrm{SI}_{\text {calcite }}$ & $\mathrm{SI}_{\text {dolomite }}$ & $\begin{array}{c}\mathrm{p}_{\mathrm{cO} 2} \\
(\mathrm{ppm})\end{array}$ & $\begin{array}{c}\delta^{13} \mathrm{C}_{\mathrm{DIC}} \\
(\%)\end{array}$ & $\begin{array}{l}\delta^{18} \mathrm{O} \\
(\% \circ)\end{array}$ & $\begin{array}{c}\delta D \\
(\% \circ) \\
\end{array}$ & $\begin{array}{c}d \\
(\% 0)\end{array}$ \\
\hline 0.47 & 0.03 & 0.62 & 2.10 & 4.58 & 2.52 & -0.39 & -1.25 & 3630.8 & -7.8 & -9.9 & -66.5 & 11.7 \\
\hline 0.06 & 0.07 & 1.27 & 1.82 & 9.91 & 10.54 & -0.34 & -1.12 & 3548.1 & -8.6 & -9.8 & -67.5 & 9.9 \\
\hline 0.78 & 0.09 & 0.36 & 1.99 & 5.24 & 1.55 & -0.01 & -0.44 & 2454.7 & -5.4 & -10.4 & -72.0 & 10.2 \\
\hline 0.81 & 0.12 & 0.71 & 3.54 & 38.87 & 1.95 & -0.04 & -0.5 & 1737.8 & -7.3 & -10.1 & -66.6 & 13.2 \\
\hline 0.29 & 0.15 & 0.74 & 3.70 & 18.45 & 4.39 & 0.58 & 0.73 & 724.4 & -7.3 & -9.8 & -67.1 & 10.3 \\
\hline 0.00 & 0.01 & 0.20 & 1.82 & 2.79 & 0.47 & 0.01 & -0.68 & 871.0 & -3.3 & -8.8 & -58.0 & 11.5 \\
\hline 0.81 & 0.05 & 1.09 & 5.39 & 5.86 & 8.42 & 0.52 & 0.28 & 2754.2 & -12.8 & -9.2 & -60.6 & 12.1 \\
\hline 0.20 & 0.02 & 0.48 & 2.81 & 7.28 & 2.91 & 0.35 & -0.01 & 1047.1 & -7.2 & -9.1 & -58.9 & 13.0 \\
\hline 1.29 & 0.11 & 0.80 & 2.88 & 14.23 & 3.55 & 0.6 & 0.74 & 871.0 & -7.3 & -9.6 & -67.5 & 8.3 \\
\hline 0.35 & 0.51 & 1.45 & 2.49 & 55.49 & 2.06 & 0.73 & 1.04 & 489.8 & -6.4 & -9.7 & -66.5 & 10.1 \\
\hline 0.47 & 0.16 & 1.28 & 3.00 & 20.14 & 2.25 & 0.53 & 0.61 & 977.2 & -7.5 & -9.3 & -63.5 & 10.0 \\
\hline 0.56 & 0.14 & 1.22 & 6.01 & 17.17 & 6.77 & 0.02 & -0.41 & 4466.8 & -8.8 & -9.3 & -68.6 & 4.9 \\
\hline 0.62 & 0.11 & 0.86 & 3.66 & 12.50 & 3.57 & 0.69 & 0.87 & 158 & -8.1 & -9.4 & -68.9 & 5.4 \\
\hline \multirow[t]{2}{*}{1.88} & 0.11 & 2.03 & 9.01 & 14.84 & 6.75 & 0.03 & -0.33 & & -10.5 & -8.8 & -57.8 & 11.7 \\
\hline & & & 4.54 & 12.07 & 3.73 & & & 7762.5 & -8.6 & -9.1 & -60.4 & 11.5 \\
\hline 6.46 & 0.07 & 1.88 & 42.60 & 17.23 & 18.11 & 0.39 & 0.29 & 4168.7 & -9.3 & -9.0 & -58.2 & 12.9 \\
\hline 2.15 & 0.11 & 1.02 & & .70 & 16.48 & 0.62 & 0.88 & 2884.0 & -11.8 & -8.7 & -58.3 & 10.4 \\
\hline 1.51 & 0.10 & 1.1 & 7.5 & 14.81 & 6.68 & 0.52 & 0.59 & 144 & -9.9 & -8.9 & -61.1 & 9.2 \\
\hline 0.98 & 0.06 & 5.46 & 1.7 & 7.35 & 3.08 & -1.51 & -3.42 & 251 & -9.0 & & -61.0 & \\
\hline 1.70 & 0.09 & 1.48 & 6.25 & 14.00 & 4.97 & -0.25 & -1.03 & 9120.1 & -11.8 & -9.0 & -65.3 & 5.8 \\
\hline \multirow[t]{2}{*}{1.78} & 0.09 & 1.53 & 7.45 & 14.74 & 6.43 & 0.27 & 0.01 & 2691.5 & -10.9 & -8.7 & -59.2 & 9.5 \\
\hline & & & 6.52 & 14.89 & 10.64 & & & 1.5 & -11.6 & & -59.5 & \\
\hline \multirow[t]{2}{*}{1.08} & 0.09 & 1.48 & 5.94 & 13.70 & 5.07 & 0.81 & 1.13 & 891.3 & -10.2 & -9.0 & -66.4 & 4.7 \\
\hline & & & 2.03 & 16.84 & 3.35 & & & 631.0 & -10.4 & -9.3 & & \\
\hline 3.67 & 0.13 & 2.97 & 7.31 & 29.78 & 9.21 & 1.31 & 2.44 & 537.0 & -11.0 & -9.4 & -63.4 & 10.9 \\
\hline 0.84 & 0.09 & 1.5 & & 14.47 & 5.52 & 0.92 & 1.35 & 977.2 & -9.8 & & -64.7 & \\
\hline 0.00 & 0.07 & 1.57 & 3. & 22.91 & 4.0 & 1.33 & 2.53 & 426.6 & -10.1 & -9.8 & -65.2 & 12.2 \\
\hline 1.02 & 0.03 & 1.07 & 4.46 & 21.70 & 2.37 & 1.24 & 2.04 & 363.1 & -10.9 & -10.1 & & \\
\hline 4.04 & 0.10 & 4.16 & 3.66 & 12.50 & 3.57 & 0.55 & 0.9 & 1905.5 & -11.4 & -9.0 & -58.0 & 13.1 \\
\hline 1.57 & 0.10 & 1.51 & 6.68 & 16.85 & 5.68 & 0.99 & 1.56 & & -10.9 & -9.1 & & \\
\hline 1.44 & 0.09 & 1.39 & 10.32 & 14.59 & 5.29 & 0.96 & 1.44 & 501.2 & -10.2 & -9.1 & -64.2 & 7.7 \\
\hline 4.72 & 0.15 & 3.78 & 6.32 & 42.22 & 14.63 & 0.87 & 1.55 & 776.2 & -11.6 & & 63.6 & \\
\hline 3.06 & 0.20 & 2.83 & & 48.86 & 11.90 & 1.25 & 2.04 & 234.4 & -10.2 & -8.8 & -63.7 & 5.8 \\
\hline 1.53 & 0.12 & 1.62 & & 22.84 & 7.50 & 0.98 & 1.51 & 467.7 & -9.9 & -8.8 & -57.0 & 12.5 \\
\hline \multirow[t]{2}{*}{3.48} & 0.12 & 2.60 & 3.2 & 17.55 & 4.92 & 1.59 & 3.01 & 234.4 & -11.2 & -9.1 & -62.1 & 9.8 \\
\hline & & & & & & & & 446.7 & -10.4 & -8.7 & -58.0 & 10.7 \\
\hline \multirow[t]{2}{*}{2.29} & 0.11 & 1.84 & 6.79 & 27.25 & 8.66 & 0.35 & 0.23 & 2290.9 & -10.8 & -8.8 & -55.7 & 13.8 \\
\hline & & & 7.27 & & & & & & -11.1 & -9.3 & -64.0 & 9.5 \\
\hline 1.91 & 0.11 & 1.79 & 6.31 & 25.83 & 8.27 & 0.71 & 0.97 & 1174.9 & -11.5 & -8.8 & -59.4 & 10.1 \\
\hline 6.77 & 0.40 & 4.52 & 8.34 & 37.39 & 13.90 & 1.41 & 2.33 & 660.7 & -12.2 & -8.5 & -60.2 & 7.0 \\
\hline 1.82 & 0.10 & 1.67 & 2.49 & 21.40 & 2.06 & 0.42 & 0.37 & 2089.3 & -10.8 & -8.9 & -59.4 & 10.9 \\
\hline
\end{tabular}

nates represent the source of inorganic material. Plants in the Sava watershed have an average value of $-31.6 \% \pm 1.5(n=22)$, while Mesozoic carbonates have an average value of $1.4 \% \pm 1.3(\mathrm{n}=13)$.

The major inputs to the DIC flux (DIC $\left.{ }_{\text {out }}\right)$ and $\delta^{13} \mathrm{C}_{\mathrm{DIC}}$ originate from tributaries $\left(\mathrm{DIC}_{\mathrm{tri}}\right)$, degradation of organic matter
$\left(\mathrm{DIC}_{\text {org }}\right)$, exchange with the atmosphere $\left(\mathrm{DIC}_{\mathrm{ex}}\right)$, and dissolution of carbonates $\left(\mathrm{DIC}_{\mathrm{ca}}\right)$ :

$$
\begin{gathered}
\mathrm{DIC}_{\text {out }}=\mathrm{DIC}_{\text {tri }}-\mathrm{DIC}_{\mathrm{ex}}+\mathrm{DIC}_{\text {org }}+\mathrm{DIC}_{\mathrm{ca}} \\
\mathrm{DIC}_{\text {out }} \cdot \delta^{13} \mathrm{C}_{\text {out }}=\mathrm{DIC}_{\mathrm{tri}} \cdot \delta^{13} \mathrm{C}_{\mathrm{tri}}-\mathrm{DIC}_{\mathrm{ex}} \cdot \\
\cdot \delta^{13} \mathrm{C}_{\mathrm{ex}}+\mathrm{DIC}_{\mathrm{org}} \cdot \delta^{13} \mathrm{C}_{\mathrm{POC}}+\mathrm{DIC}_{\mathrm{ca}} \cdot \delta^{13} \mathrm{C}_{\mathrm{Ca}}
\end{gathered}
$$


Table 3. Chemical and isotopic data for the River Sava watershed, winter 2005. Locations are plotted on Figure 1.

\begin{tabular}{|c|c|c|c|c|c|c|c|c|c|c|}
\hline $\begin{array}{l}\text { Sampling } \\
\text { point }\end{array}$ & $\begin{array}{c}Q \\
\left(\mathrm{~m}^{3} / \mathrm{s}\right)\end{array}$ & $\begin{array}{c}\mathrm{T} \\
\left({ }^{\circ} \mathrm{C}\right)\end{array}$ & $\begin{array}{c}\text { Conductivity } \\
(\mu \mathrm{S} / \mathrm{cm})\end{array}$ & $\mathrm{pH}$ & $\begin{array}{l}\text { Alkalinity } \\
\text { (mmo/l) }\end{array}$ & $\begin{array}{l}\mathrm{DOC} \\
(\mathrm{mg} / \mathrm{l})\end{array}$ & $\begin{array}{c}\mathrm{Ca} \\
(\mathrm{mg} / \mathrm{l})\end{array}$ & $\underset{(\mathrm{mg} / \mathrm{l})}{\mathrm{Mg}}$ & $\begin{array}{c}\mathrm{Na} \\
(\mathrm{mg} / \mathrm{l})\end{array}$ & $\begin{array}{c}\mathrm{K} \\
(\mathrm{mg} / \mathrm{l})\end{array}$ \\
\hline 1 & & 5.3 & 257 & 8.06 & 2.73 & & 39.21 & 11.78 & 1.21 & 0.70 \\
\hline 2 & 0.66 & 2.3 & 286 & 8.25 & 2.67 & 0.84 & 41.78 & 12.39 & 2.33 & 0.58 \\
\hline 3 & & 2.3 & 285 & 8.29 & 2.35 & 1.26 & 35.54 & 10.92 & 0.85 & 1.20 \\
\hline 4 & 6.66 & 5.1 & 288 & 8.34 & 2.88 & 1.04 & 43.80 & 12.94 & 1.24 & 0.96 \\
\hline 5 & 14.90 & 3.3 & 327 & 8.43 & 3.14 & 0.77 & 51.54 & 14.13 & 2.69 & 0.29 \\
\hline 6 & & 3 & 187 & 8.51 & 1.81 & & & & & \\
\hline 7 & 1.40 & 3.3 & 187 & 8.25 & 2.12 & 0.93 & & & & \\
\hline 8 & 2.95 & 3.2 & 260 & 8.75 & 2.73 & & 48.20 & 6.91 & 0.61 & 0.43 \\
\hline 9 & 20.70 & 3.1 & 391 & 8.21 & 3.42 & 1.03 & 56.68 & 13.53 & 2.57 & 3.88 \\
\hline 10 & 2.84 & 3 & 385 & 8.42 & 2.48 & 1.02 & 69.20 & 26.57 & 3.13 & 0.53 \\
\hline 11 & 1.69 & 2.4 & 337 & 8.51 & 3.18 & 3.07 & 57.42 & 14.13 & 1.62 & 3.98 \\
\hline 12 & 9.69 & 4.2 & 440 & 8.02 & 3.64 & 0.24 & 62.17 & 14.38 & 8.06 & 1.83 \\
\hline 13 & 92.30 & 4.4 & 373 & 8.1 & 3.51 & 0.54 & 64.08 & 15.91 & 5.72 & 1.96 \\
\hline 14 & 7.03 & 3.6 & 334 & 8.22 & 3.11 & 0.77 & 51.99 & 14.45 & 3.00 & 0.92 \\
\hline 15 & & 4.5 & 373 & 8.26 & 3.44 & & 58.19 & 14.45 & 3.41 & 0.88 \\
\hline 16 & 0.31 & 7.7 & 565 & 7.76 & 4.89 & 1.66 & 79.33 & 16.64 & 14.17 & 4.31 \\
\hline 17 & 18.10 & 5.5 & 401 & 8.14 & 3.95 & 0.79 & 70.43 & 15.66 & 2.21 & 0.48 \\
\hline 18 & & 5.2 & 415 & 8.12 & 3.82 & 0.89 & 62.78 & 15.01 & 4.30 & 0.52 \\
\hline 19 & & 1.6 & & 7.85 & 0.44 & 1.43 & 6.76 & 1.89 & 2.56 & 0.80 \\
\hline 20 & & 3.8 & 414 & 8.25 & & & & & & \\
\hline 21 & & 4.3 & 441 & 8.31 & 4.00 & 1.64 & 66.01 & 14.92 & 4.32 & 1.13 \\
\hline 22 & & 2.4 & & 8 & 3.71 & 0.11 & 46.76 & 23.54 & 2.92 & 0.52 \\
\hline 23 & & 1.9 & 423 & 7.98 & 3.93 & 1.44 & 64.11 & 15.48 & 5.03 & 2.64 \\
\hline 24 & & 2.1 & & 8.1 & 2.24 & 0.48 & 28.12 & 14.37 & 2.46 & 2.33 \\
\hline 25 & & 1.4 & & 8.17 & 4.62 & 0.24 & 69.74 & 23.65 & 6.07 & 1.47 \\
\hline 26 & & 2.8 & & 7.88 & 3.58 & 0.13 & 63.57 & 15.82 & 5.33 & 3.64 \\
\hline 27 & & 1.6 & & 8.38 & 4.71 & 0.86 & 62.66 & 27.89 & 1.94 & 2.87 \\
\hline 28 & & 5.5 & 370 & 8.28 & 3.58 & 2.15 & 63.04 & 10.96 & 0.41 & 0.78 \\
\hline 29 & & 4.4 & 928 & 7.87 & 6.30 & & & & & \\
\hline 30 & & 3.2 & 432 & 7.94 & 4.22 & 0.60 & 64.68 & 15.73 & 5.35 & 1.44 \\
\hline 31 & 62.20 & 3.9 & 421 & 7.97 & 4.17 & 0.40 & 69.53 & 9.35 & 0.47 & 2.31 \\
\hline 32 & & 3.2 & 544 & 8.1 & 4.89 & 1.55 & 63.67 & 24.39 & 12.96 & 3.88 \\
\hline 33 & 12.40 & 1.3 & 481 & 8.54 & 3.80 & & 72.08 & 14.58 & 13.82 & 3.44 \\
\hline 34 & & 3.4 & 444 & 8.14 & 3.88 & 0.42 & 60.01 & 14.06 & 3.71 & 1.10 \\
\hline 35 & 1.29 & & & & 5.10 & & & & & 0.00 \\
\hline 36 & & 3.2 & 424 & 8.2 & 3.97 & 0.53 & 65.21 & 15.63 & 5.96 & 1.30 \\
\hline 37 & & 6.2 & 467 & 8.08 & 3.96 & 0.35 & 72.76 & 16.71 & 6.67 & 1.49 \\
\hline 38 & 12.40 & 2.8 & 469 & 8.44 & 4.33 & & & & & \\
\hline 39 & 96.30 & 5.4 & 445 & 8.27 & 3.54 & 0.55 & 67.21 & 16.11 & 6.71 & 1.64 \\
\hline 40 & 1.67 & 0.4 & 606 & 8.22 & 5.91 & 0.84 & 96.95 & 22.18 & 12.49 & 4.14 \\
\hline 41 & 128.00 & 4.9 & 445 & 8.29 & 3.49 & 0.53 & 69.17 & 16.57 & 5.79 & 1.83 \\
\hline
\end{tabular}

The process of photosynthesis was considered insignificant and therefore excluded from the mass balance calculations. Dissolved oxygen saturation reached a maximum of only $118 \%$ in the late summer sampling and the $\delta^{13} \mathrm{C}_{\mathrm{POC}}$, with an average value of $-26.7 \%$, indicates that organic matter derived from aquatic photosynthesis is re- latively insignificant in the River Sava system.

According to the concentration and isotope mass balance calculations (equations 2 and 3 ) of dissolved inorganic carbon, the proportions of the four processes (1) effect of tributaries $\left(\mathrm{DIC}_{\mathrm{trb}}\right),(2)$ degradation of organic matter $\left(\mathrm{DIC}_{\text {org }}\right)$, (3) dissolution of 


\begin{tabular}{|c|c|c|c|c|c|c|c|c|c|c|c|}
\hline $\mathrm{Sr}$ (mg/l) & $\begin{array}{c}\mathrm{Si} \\
(\mathrm{mg} / \mathrm{l})\end{array}$ & $\begin{array}{c}\mathrm{NO}_{3} \\
(\mathrm{mg} / \mathrm{l})\end{array}$ & $\begin{array}{c}\mathrm{SO}_{4} \\
(\mathrm{mg} / \mathrm{l})\end{array}$ & $\begin{array}{c}\mathrm{Cl} \\
(\mathrm{mg} / \mathrm{l})\end{array}$ & $\mathrm{SI}_{\text {calcite }}$ & $\mathrm{SI}_{\text {dolomite }}$ & $\begin{array}{c}\mathrm{p}_{\mathrm{cO} 2} \\
(\mathrm{ppm})\end{array}$ & $\begin{array}{c}\delta^{13} \mathrm{C}_{\mathrm{DIC}} \\
(\% 0)\end{array}$ & $\delta^{18} \mathrm{O}(\% 0)$ & $\begin{array}{c}\delta D \\
(\%)\end{array}$ & $\begin{array}{c}d \\
(\% 0)\end{array}$ \\
\hline 0.00 & 0.25 & 3.44 & 4.34 & 2.78 & 0.17 & -0.16 & 1174.9 & -9.3 & -9.1 & -70 & 2.3 \\
\hline 0.06 & 0.65 & 3.40 & 11.59 & 5.52 & 0.32 & 0.07 & 707.9 & -8.2 & -10.0 & & \\
\hline 0.08 & 0.17 & 1.77 & 7.42 & 2.21 & 0.24 & -0.07 & 575.4 & -7.3 & & & \\
\hline 0.11 & 0.34 & 2.22 & 14.56 & 3.06 & 0.50 & 0.49 & 631.0 & -7.5 & -10.0 & -69 & 9.7 \\
\hline \multirow[t]{3}{*}{0.16} & 0.48 & 3.00 & 20.81 & 5.75 & 0.65 & 0.73 & 549.5 & -6.3 & -9.8 & -68 & 9.3 \\
\hline & & 1.70 & 2.53 & 0.45 & & & 263.0 & -4.2 & -9.6 & -59 & 17.1 \\
\hline & & 2.18 & 2.91 & 0.96 & & & 575.4 & -7.5 & -9.6 & -64 & 12.3 \\
\hline 0.01 & 0.25 & 2.86 & 4.56 & 1.92 & 0.87 & 0.90 & 223.9 & -7.3 & -8.8 & -59 & 10.3 \\
\hline 0.09 & 0.50 & 6.14 & 16.30 & 9.68 & 0.51 & 0.38 & 1000.0 & -8.9 & -9.1 & -63 & 9.1 \\
\hline 0.09 & 0.90 & 3.38 & 52.98 & 5.01 & & & 446.7 & -5.8 & -9.6 & -67 & 9.3 \\
\hline 0.45 & 0.60 & 1.77 & 12.90 & 1.31 & & & 457.1 & -6.4 & -9.1 & -61 & 10.8 \\
\hline 0.14 & 0.61 & 8.31 & 23.06 & 12.58 & 0.40 & 0.17 & 1659.6 & -9.4 & -9.3 & -63 & 10.5 \\
\hline 0.10 & 0.53 & 7.77 & 20.96 & 6.80 & & & 1349.0 & -8.7 & -9.1 & -61 & 10.8 \\
\hline 0.07 & 1.03 & 8.21 & 14.04 & 4.77 & 0.45 & 0.35 & 891.3 & -9.7 & -8.9 & -59 & 11.4 \\
\hline 0.12 & 0.67 & 8.96 & 21.57 & 7.25 & 0.59 & 0.59 & 912.0 & -8.5 & -9.2 & -63 & 10.0 \\
\hline 0.06 & 0.93 & 39.21 & 19.23 & 19.94 & 0.41 & 0.22 & 4265.8 & -10.9 & & & \\
\hline 0.06 & 0.58 & 5.22 & 13.15 & 4.68 & & & 1380.4 & -11.9 & -8.6 & -60 & 7.5 \\
\hline 0.09 & 0.68 & 8.21 & 16.94 & 7.00 & 0.54 & 0.48 & 1412.5 & -10.0 & -8.9 & -59 & 10.9 \\
\hline 0.01 & 2.32 & 2.52 & 6.92 & 2.61 & -1.57 & -3.74 & 302.0 & $\begin{array}{l}\text { n.a. } \\
\text { n.a. }\end{array}$ & -8.8 & -61 & 8.3 \\
\hline 0.09 & 0.65 & & & & & & 933.3 & -9.7 & -8.8 & -61 & 8.7 \\
\hline 0.01 & 1.23 & 4.29 & 13.28 & 4.59 & 0.24 & 0.14 & 1737.8 & -10.7 & -8.7 & -63 & 5.7 \\
\hline 0.10 & 0.50 & 9.81 & 19.39 & 10.30 & 0.37 & 0.07 & 1949.8 & -10.0 & -8.3 & -60 & 5.3 \\
\hline 0.03 & 1.73 & 2.17 & 16.47 & 3.71 & -0.07 & -0.47 & 851.1 & -10.4 & -8.9 & -57 & 13.7 \\
\hline 0.11 & 1.01 & 5.06 & 30.37 & 7.86 & 0.63 & 0.74 & 1445.4 & -10.2 & -9.1 & -63 & 9.1 \\
\hline 0.10 & 0.54 & 8.29 & 19.76 & 10.54 & 0.24 & -0.14 & 2238.7 & -9.1 & -8.9 & -61 & 9.5 \\
\hline 0.04 & 0.57 & 3.73 & 25.96 & 6.24 & 0.80 & 1.20 & 912.0 & -9.0 & -9.3 & -66 & 8.0 \\
\hline \multirow[t]{2}{*}{0.00} & 0.45 & 4.45 & 18.66 & 3.16 & 0.68 & 0.63 & 912.0 & -10.6 & -9.8 & -62 & 15.4 \\
\hline & & & & & & & 4073.8 & -11.0 & -9.1 & -63 & 8.9 \\
\hline 0.11 & 0.54 & 9.13 & 21.59 & 8.98 & 0.38 & 0.13 & 2290.9 & -9.5 & -8.8 & -56 & 13.1 \\
\hline 0.00 & 0.44 & 8.11 & 19.97 & 8.44 & & & 2138.0 & -9.5 & -8.9 & -63 & 7.2 \\
\hline 0.10 & 1.34 & 9.05 & 40.60 & 15.85 & 0.58 & 0.73 & 1819.7 & -8.5 & -9.4 & -62 & 12.3 \\
\hline 0.18 & 1.02 & 10.70 & 44.57 & 14.08 & 0.93 & 1.10 & 501.2 & -8.8 & -9.1 & -62 & 9.6 \\
\hline 0.13 & 0.61 & 9.08 & 23.00 & 9.65 & 0.52 & 0.40 & 1349.0 & -10.5 & -8.8 & -61 & 9.0 \\
\hline 0.00 & 0.00 & 3.83 & 18.00 & 5.63 & & & & -10.0 & -9.2 & -62 & 11.0 \\
\hline 0.11 & 0.65 & 8.54 & 21.66 & 8.99 & 0.61 & 0.59 & 1174.9 & -9.2 & -8.9 & -62 & 8.1 \\
\hline \multirow[t]{2}{*}{0.12} & 0.76 & 9.10 & 25.63 & 10.18 & 0.59 & 0.58 & 1621.8 & -10.6 & -8.9 & -60 & 10.1 \\
\hline & & 8.09 & 10.07 & 6.33 & & & 741.3 & -10.3 & -9.8 & -65 & 12.5 \\
\hline 0.11 & 0.63 & 10.40 & 23.60 & 9.97 & & & 912.0 & -10.4 & -8.8 & -61 & 8.2 \\
\hline 0.37 & 1.28 & 5.55 & 38.90 & 16.04 & 0.75 & 0.93 & 1621.8 & -10.9 & -9.4 & -67 & 7.1 \\
\hline 0.10 & 0.62 & 5.47 & 15.15 & 6.68 & & & 1096.5 & -9.8 & -9.2 & -60 & 12.8 \\
\hline
\end{tabular}

carbonates (DIC $\mathrm{Darb}_{\text {) }}$ ) and (4) equilibration of atmospheric $\mathrm{CO}_{2}\left(\mathrm{DIC}_{\mathrm{ex}}\right)$ that influence $\delta^{13} \mathrm{C}_{\mathrm{DIC}}$ values in the river were calculated. The calculated proportions of each process, i. e. $\mathrm{DIC}_{\text {trb }}$ : $\mathrm{DIC}_{\mathrm{org}}$ : $\mathrm{DIC}_{\mathrm{ex}}$ : $\mathrm{DIC}_{\text {carb }}$ vary seasonally and at Bregana (41) are: $70: 11$ : $-1: 19 \%$ in spring 2004, 62: $17:-5: 26 \%$ in late summer 2004 and $70: 11:-4: 23 \%$ in winter 2005. Among the biogeochemical processes the dissolution of carbonates contributes a higher proportion than degradation of organic matter and is reflected in all sampling seasons (Kanduč, 2006, Kanduč et al. in press). 

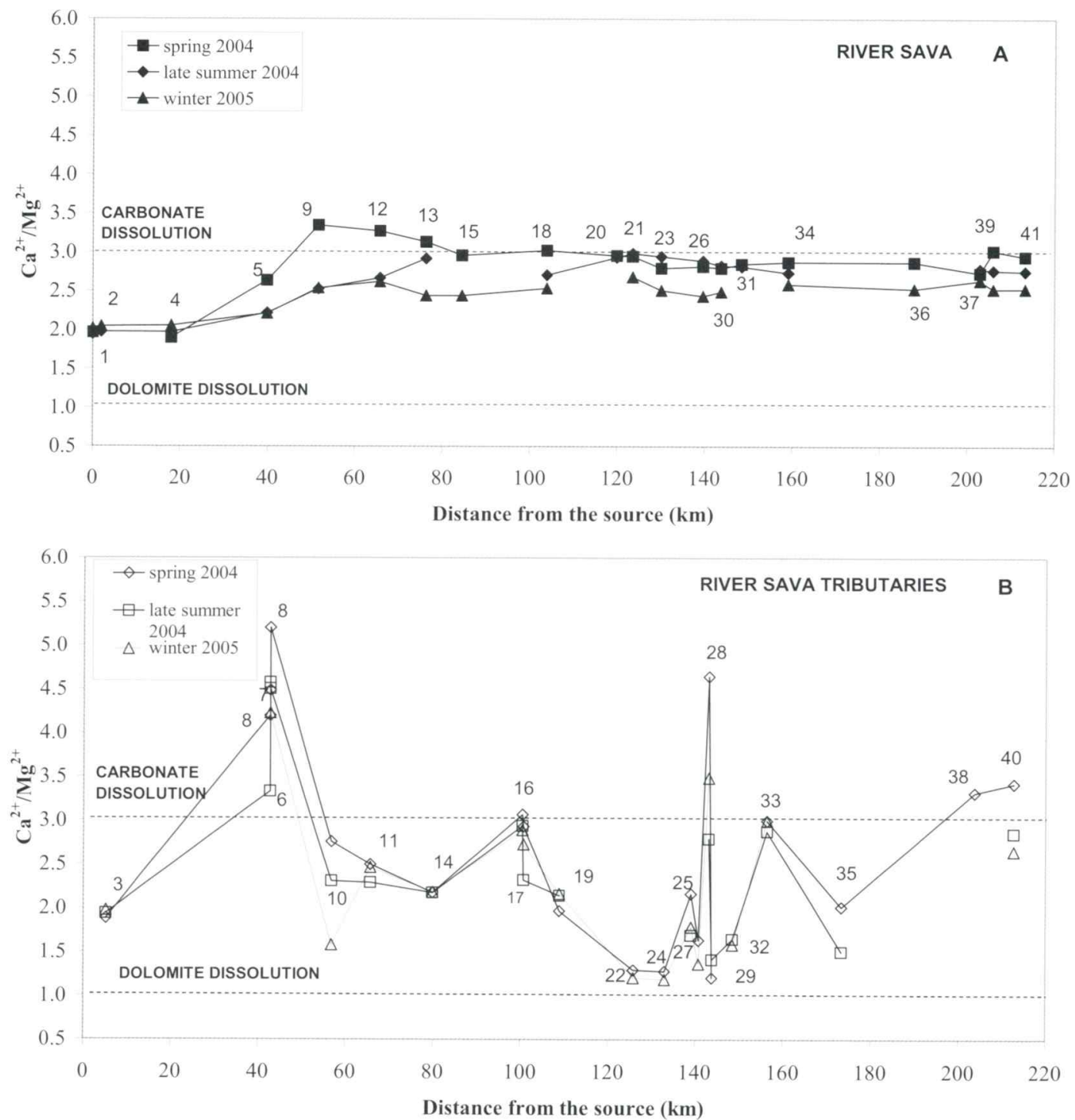

Figure 2. $\mathrm{Ca}^{2+} / \mathrm{Mg}^{2+}$ ratio versus distance to the source (A - River Sava, B - River Sava tributaries).

\section{Oxygen and Hydrogen Isotopes}

Discharges at the sampling locations (Tables 1, 2 and 3) in the River Sava are dependent on precipitation, evaporation, evapotranspiration, infiltration, equilibration with run-off and anthropogenic engineering structures like dams (Yee et al., 1990).

The contributions of tributary discharges to the River Sava were estimated at locations where discharge data exists (Tables 1, 2 and 3 ). At the confluence of the Sava Bohinjka and Sava Dolinka (sampling point 9,
Sava Otoče) the Sava Bohinjka contributes $64 \%$, while the Sava Dolinka represents $55 \%$ of the total discharge at the confluence in the spring sampling season. In the late summer and winter sampling season the Sava Bohinjka contributes 26 and $14 \%$ of total discharge, while the Sava Dolinka contributes $72 \%$. The tributaries have higher contributions in the spring sampling season due to snow melting, the higher amount of precipitation and consequent surface and subsurface leaching from the slopes to the tributaries and finally to rivers. Those Sava 

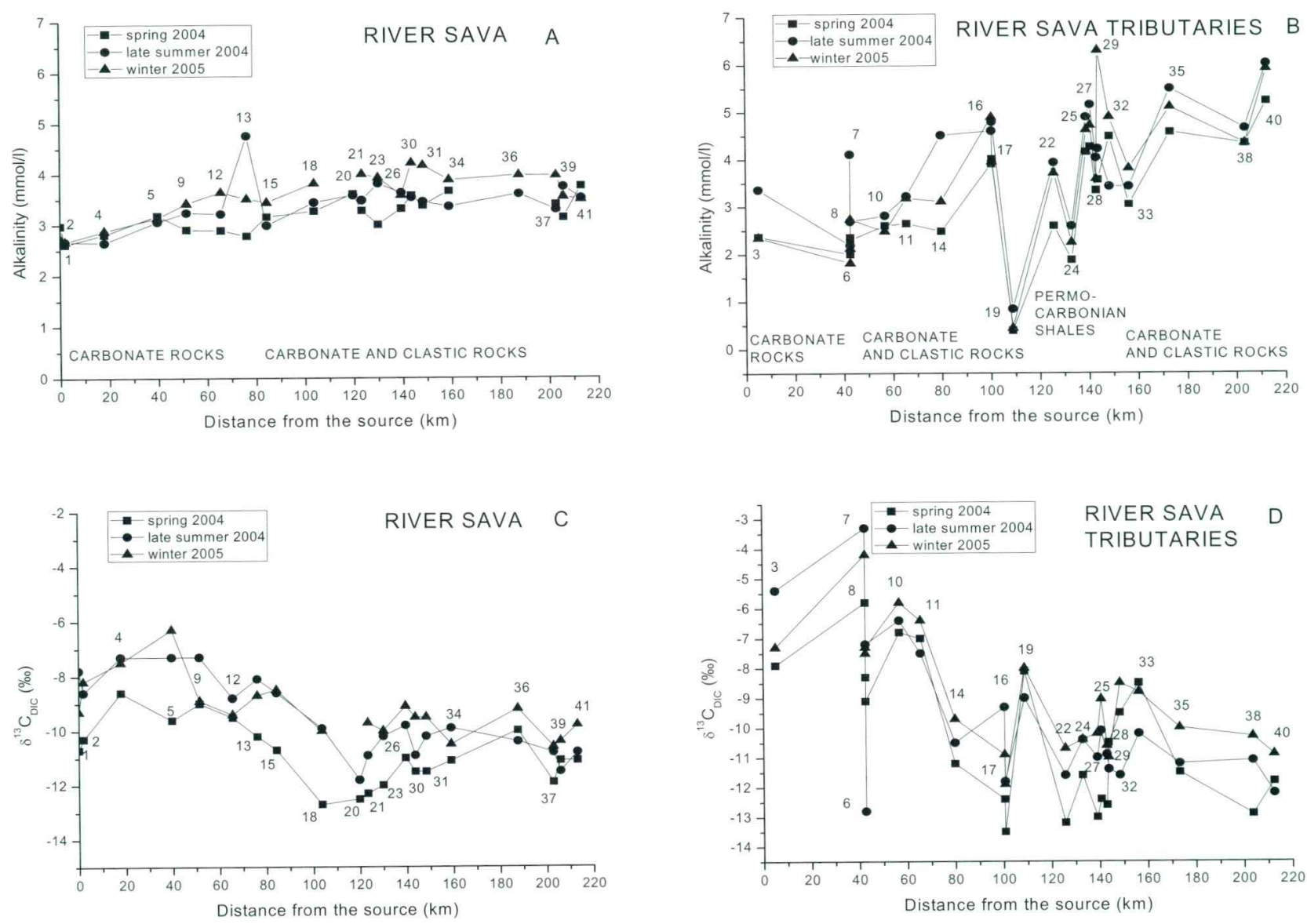
tributaries with higher drainage areas, namely the Ljubljanica (17), Savinja (33) and Krka (38), have contributions of $42 \%$, $14 \%$ and $18 \%$ in the spring sampling season, $7 \%, 27 \%$ and $22 \%$ in late summer and $33 \%, 20 \%$ and $13 \%$ in winter, respectively.

$\delta^{18} \mathrm{O}_{\text {water }}$ values in the River Sava seasonally changed from -11.0 to $-9.7 \%$ in spring 2004, from -10.1 to $-8.9 \%$ in late summer 2004 and from -10.0 to $-8.8 \%$ in winter 2006, respectively. Values of $\delta^{18} \mathrm{O}_{\text {water }}$ of Sava tributaries varyed from - 12.4 to - $9.1 \%$ in spring 2004, from - 10.4 to $-8.7 \%$ in late summer 2004 and from -9.8 to $-8.6 \%$ in winter month (Tables 1, 2 and 3, Figure $4 \mathrm{~A}$ and $4 \mathrm{~B}$ ). Lower $\delta^{18} \mathrm{O}_{\text {water }}$ was observed in the Sava watershed in spring 2004 while in the late summer and winter seasons lower $\delta^{18} \mathrm{O}$ values were observed due to the high amount of precipitation and water leached from slopes forming the watershed (Vreča et al., 2004). In the winter sampling season more negative $\delta^{18} \mathrm{O}$ values would be expected due to the isotopically lighter precipitation, but in fact the values were similar to the late summer sampling season. Seasonal variation of $\delta^{18} \mathrm{O}$ values in the Sava watershed results from differences in water temperature and the height of the recharge area between sampling points in the watershed. The River water temperature is higher in all three seasons in the central and lower part of the River Sava flow in comparison with the upper part of the flow (sampling point 9). The height of the recharge area in the upper flow (sampling point 5) is above $1500 \mathrm{~m}$ a. s. 1 . in comparison to the central and lower part of the flow, where the height above sea level is up to $1000 \mathrm{~m} \mathrm{a}$. s. 1 .

The longer residence time in dams could affect the evaporation process and enrichment with heavier oxygen isotope in the summer months in the lower part of the Sava flow (location 36, see Figure $4 \mathrm{~A}$ and 4 B). Kinetic effects during evaporation may be attributed to surface water temperature, wind velocity (shear at the surface water) and, most importantly the relative humidity of the air (molecular diffusion between water-atmosphere) (Gonfiantini, 1986). Evaporation between the River Sava and the atmosphere in the watershed is also dependent on the air temperature, which varied from 8.6 to $14.2^{\circ} \mathrm{C}$ in spring 2004 , from 10 to $19{ }^{\circ} \mathrm{C}$ in late summer 2004 and from -4.9 to $2.2^{\circ} \mathrm{C}$ in winter (EIONET, 2005). In the Sava watershed fog phenomena (condensation), which are more pronounced in late summer, could also daily affect $\delta^{18} \mathrm{O}_{\text {water }}$ and $\delta \mathrm{D}_{\text {water }}$ values (Petkovšek, 1969).

In the spring sampling season the $\delta^{18} \mathrm{O}$ value was lower above the confluence of the Sava Dolinka and Sava Bohinjka (sampling location 5) than below the confluence (sampling location 9) since the Sava Bohinjka (sampling location 8) contributes $64 \%$ of the discharge, with higher $\delta^{18} \mathrm{O}_{\text {water }}$ than the Sava Dolinka. A higher deviation in $\delta^{18} \mathrm{O}_{\text {water }}$ values between the seasons was observed at sampling location 6 (Sava Bohinjka source). In spring the lower $\delta^{18} \mathrm{O}_{\text {water }}$ value is due to snow melting, isotopically lighter rain and the height of the recharge area, which is around $1800 \mathrm{~m}$ a. s. 1. (Urbanc \& Brancelj, 2002). The higher $\delta^{18} \mathrm{O}_{\text {water }}$ value at location 6 in comparison to other locations could be due to isotopically heavier precipitation in the late summer months since generation of water vapour and precipitation is a complicated process, which includes kinetic evaporation and mixing (Clark \& Fritz, 1997). The lower $\delta^{18} \mathrm{O}$ at sampling location (7), which reflects mixing of water and biogeochemical processes in lake Bohinj in the late summer season in comparison with sampling location (8), could be attributed to mixing of waters in the lake after stratification (mixing of cold and warm water) and/or anthropogenic influence. In the late summer season at sampling location (7) higher concentrations of other parameters were also observed $\left(\mathrm{Ca}^{2+}, \mathrm{Mg}^{2+}, \mathrm{HCO}_{3}^{-}, \mathrm{Na}^{+}, \mathrm{K}^{+}, \mathrm{SO}_{4}{ }^{2-}\right.$ and $\mathrm{NO}_{3}^{-}$).

$\delta \mathrm{D}_{\text {water }}$ values in the Sava River watershed ranged from -80.0 to $-55.7 \%$ in spring 2004 , from -72.0 to $-55.7 \%$ in late summer 2004 and from $-69.6 \%$ to $-56.4 \%$ in winter 2005 , respectively. The correlation between $\delta^{18} \mathrm{O}_{\text {water }}$ and $\delta \mathrm{D}_{\text {water }}$ for most meteorological and surface waters which do not reflect evaporation is expressed by the GMWL (Global Meteoric Water Line), which is determined by the equation: $\delta \mathrm{D}=8 \cdot \delta^{18} \mathrm{O}+10$ and is valid for a humidity of more than $85 \%$ (Clark and Fritz, 1997). In precipitation from Ljubljana the local meteorological line (LMWL) for the period from 2001 to 2003 was expressed by $\delta \mathrm{D}=7.9 \delta^{18} \mathrm{O}+8.3$ (Vreča et al., 2004). Sampling points deviating from the LMWL in the Sava watershed are a result of kinetic evaporation/condensation processes and are dependent on conditions above the surface water (temperature, 

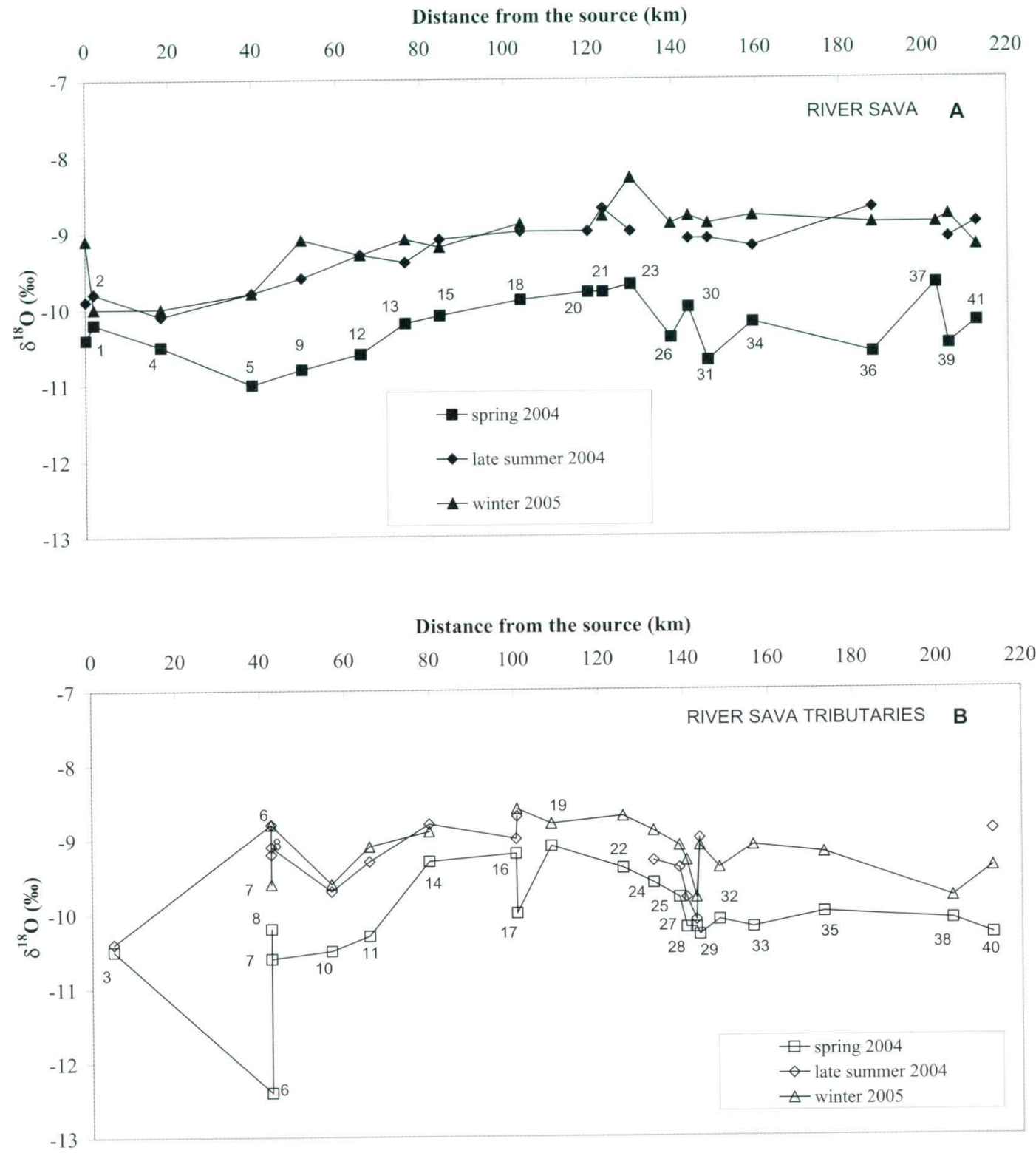

Figure 4. $\delta^{18} \mathrm{O}$ versus distance to the source of the River Sava (A - River Sava, B - River Sava tributaries).

relative humidity). Previous investigations of the isotopic composition of precipitation collected in the Mediterranean basin indicate a deuterium excess (d) for the western part of the Mediterranean basin of $14 \%$, while for eastern part it was $22 \%$ (IAEA, 2005). It was found that at locations where the value of $d$ in precipitation is below $10 \%$ (Dansgaard, 1964) this is due to secondary processes (evaporation in periods of low relative humidity in the atmosphere), while values above $10 \%$ belong to precipitation from the Mediterranean Sea (Cruz San et al., 1992). In the Sava watershed d values changed from 9.1 to $21.4 \%$ in spring 2004 , from 4.7 to $13.8 \%$ in late summer 2004 and from 2.3 to $17.1 \%$ in winter 2005 (Tables 1, 2 and 3). 


\section{Sulphur isotopes}

The concentration of sulphate in the Sava watershed changed from 6 to $39.6 \mathrm{mg} / \mathrm{l}$ in spring 2004, from 4.6 to $55.5 \mathrm{mg} / \mathrm{l}$ in late summer and from 4.3 to $25.6 \mathrm{mg} / \mathrm{l}$ in winter (Tables 1, 2 and 3, Figure 6). The highest concentrations were observed in the periods of low discharges (late summer 2004) while the opposite trend is observed in spring ( $\mathrm{Fi}-$ gure 6). The lowest sulphate concentration was observed at the Sava Dolinka spring (sampling point 1, Figure 1) during different sampling seasons. The highest sulphate concentrations were observed in all sampling seasons at the locations Trboveljščica (29), Savinja (33) and Sotla (40) due to anthropogenic influences, arising from coal mining waste water at location 29 , industry with sulphuric acid production at location 33 and spraying vinyards with Bordeaux mixture at location 40. High sulphate concentrations were also observed at Tržiška Bistrica (10) due to dissolution of Palaeozoic carbonates with evaporates which form the watershed. Furthermore, higher concentrations of sulphate were observed in the River Sava at sampling locations 4 and 26. At all sampling locations in the Sava River watershed sulphate concentrations are below $250 \mathrm{mg} / \mathrm{l}$, which represents the limiting concentration for tap water.
Values of $\delta^{34} \mathrm{~S}$ in the Sava watershed (Figure 7) which were sampled in spring 2004 varied from - $1.3 \%$ (sampling point 40 ) to $14.9 \%$ (sampling point 10). More positive values (14.9\%, sampling point 10) indicate dissolution of evaporates (Krouse, 1980). The $\delta^{34} \mathrm{~S}$ depleted signature of the River Sotla suggests that its $\mathrm{SO}_{4}{ }^{2-}$ was derived from the oxidation of sulphide minerals. Oxidation of sulphide minerals composing sedimentary clastic rocks could contribute higher sulphate concentrations than atmospheric sources (precipitation) and result in $\delta^{34} \mathrm{~S}_{\mathrm{SO} 4}$ values up to $10 \%$ (Clark \& Fritz, 1997). Figure 7 also shows that most of the samples did not originate from precipitation (except sampling point 1) and from soil (except locations 11, 16, 36 and 39) due to their higher concentrations of sulphate. Precipitation and soil water are not the sole sources contributing to sulphate concentrations in Sava water samples.

Assuming that the sources of $\mathrm{SO}_{4}{ }^{2-}$ to the Sava River are from tributaries $\left(\mathrm{F}_{\mathrm{tri}}\right)$, precipitation $\left(\mathrm{F}_{\mathrm{p}}\right)$ and other sources $\left(\mathrm{F}_{\text {other }}\right)$, the contributions of these inputs can be quantified by the following steady state equations:

$$
\begin{aligned}
\mathrm{F}_{\mathrm{SR}}=\mathrm{F}_{\mathrm{tri}} & +\mathrm{F}_{\mathrm{p}}+\mathrm{F}_{\text {other }} \\
\delta^{34} \mathrm{~S}_{\mathrm{SR}} \cdot \mathrm{F}_{\mathrm{SR}} & =\delta^{34} \mathrm{~S}_{\text {tri }} \mathrm{F}_{\text {tri }}+\delta^{34} \mathrm{~S}_{\mathrm{p}} \mathrm{F}_{\mathrm{p}}+ \\
& +\delta^{34} \mathrm{~S}_{\text {other }} \mathrm{F}_{\text {other }}
\end{aligned}
$$

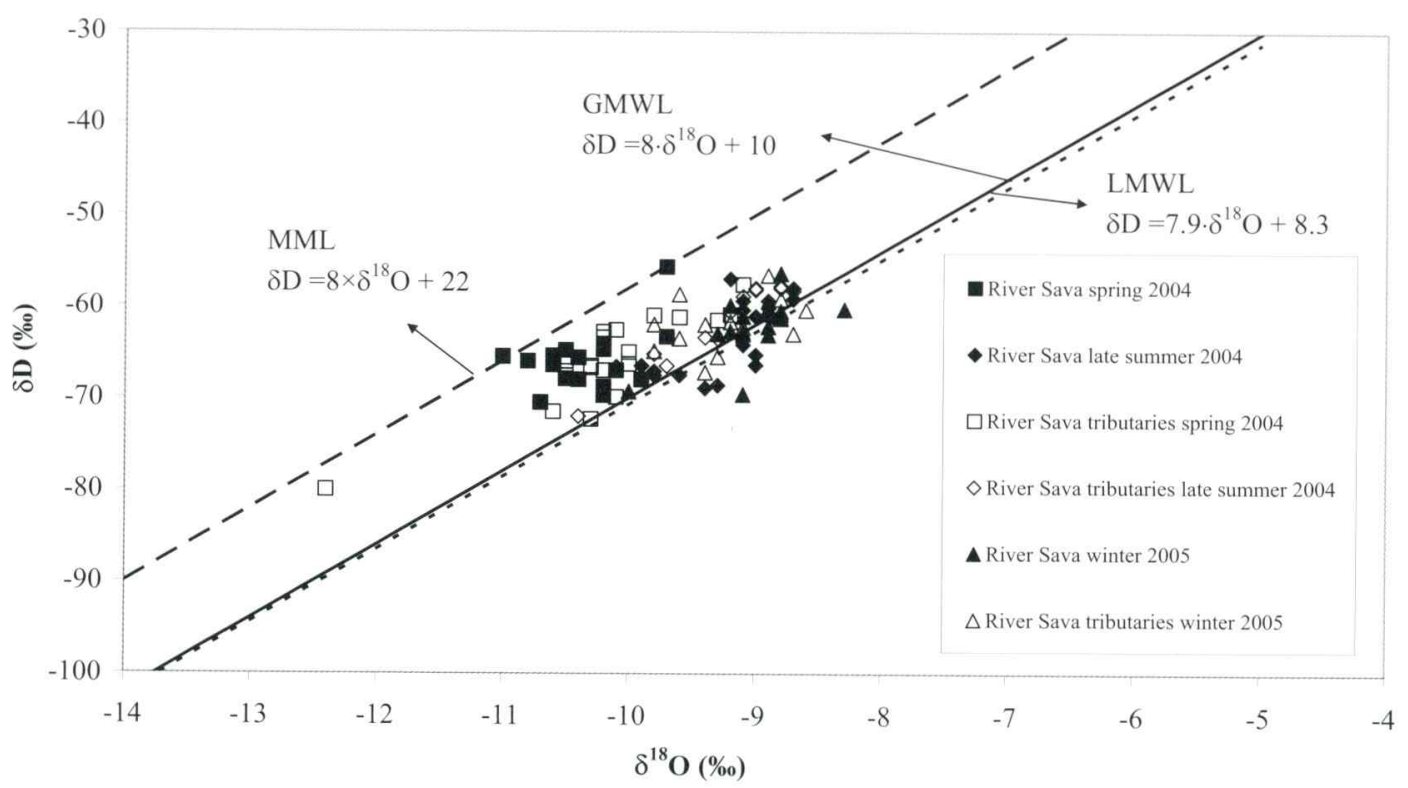

Figure 5. $\delta \mathrm{D}$ vs. $\delta^{18} \mathrm{O}$ for the River Sava system in Slovenia. GMWL - Global meteorological water line, LMWL - Local meteorological water line, MML - Mediterranean meteorological water line. 

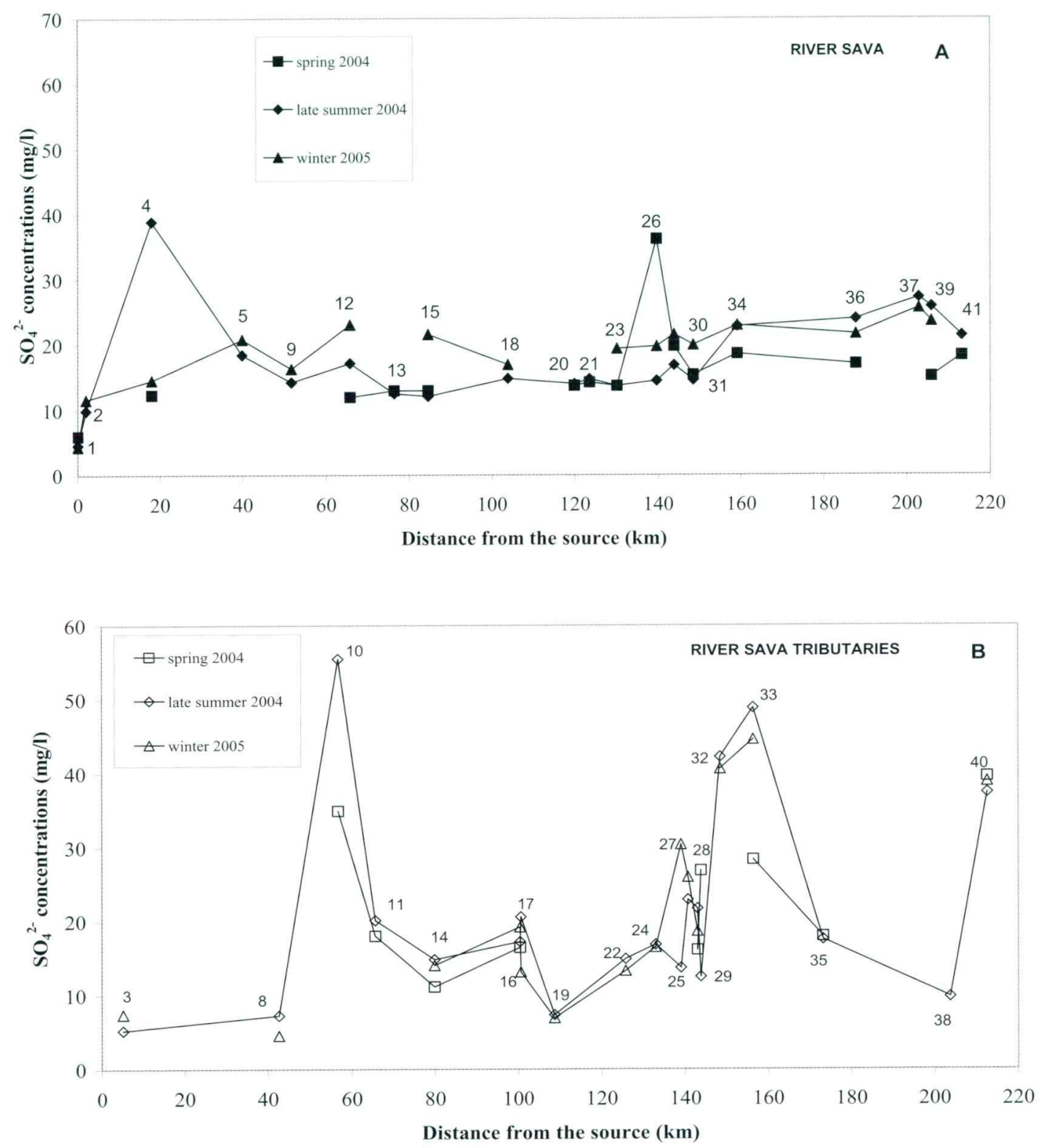

Figure 6. Sulphate concentrations versus distance to the source (A - River Sava, B - River Sava tributaries).

Where:

$$
\mathrm{Fi}=\mathrm{Qi} \cdot\left[\mathrm{SO}_{4}^{2-}\right]_{\mathrm{i}}(\mathrm{mol} / \mathrm{s})
$$

$\mathrm{i}=\mathrm{RS}$ - River Sava, tri - tributaries, $\mathrm{p}$ - precipitation, other - other sources

$\mathrm{F}_{\mathrm{RS}}(\mathrm{mol} / \mathrm{s})$ - is the annual dissolved sulphate flow at the Bregana sampling location and contributions affecting flow of dissolved sulphate in water: from tributaries $\left(\mathrm{F}_{\text {tri }}\right)$, precipitation $\left(\mathrm{F}_{\mathrm{p}}\right)$ and other sources $\left(\mathrm{F}_{\text {other }}\right)$ $\delta^{34} \mathrm{~S}_{\mathrm{RS}}(\%)$ - is the measured isotopic composition of sulphur at the Bregana sampling location and contributions affecting isotopic composition of sulphate at this point: from tributaries $\left(\delta^{34} \mathrm{~S}_{\mathrm{tri}}\right)$, precipitation $\left(\delta^{34} \mathrm{~S}_{\mathrm{p}}\right)$ and other sources $\left(\delta^{34} \mathrm{~S}_{\text {other }}\right)$ 


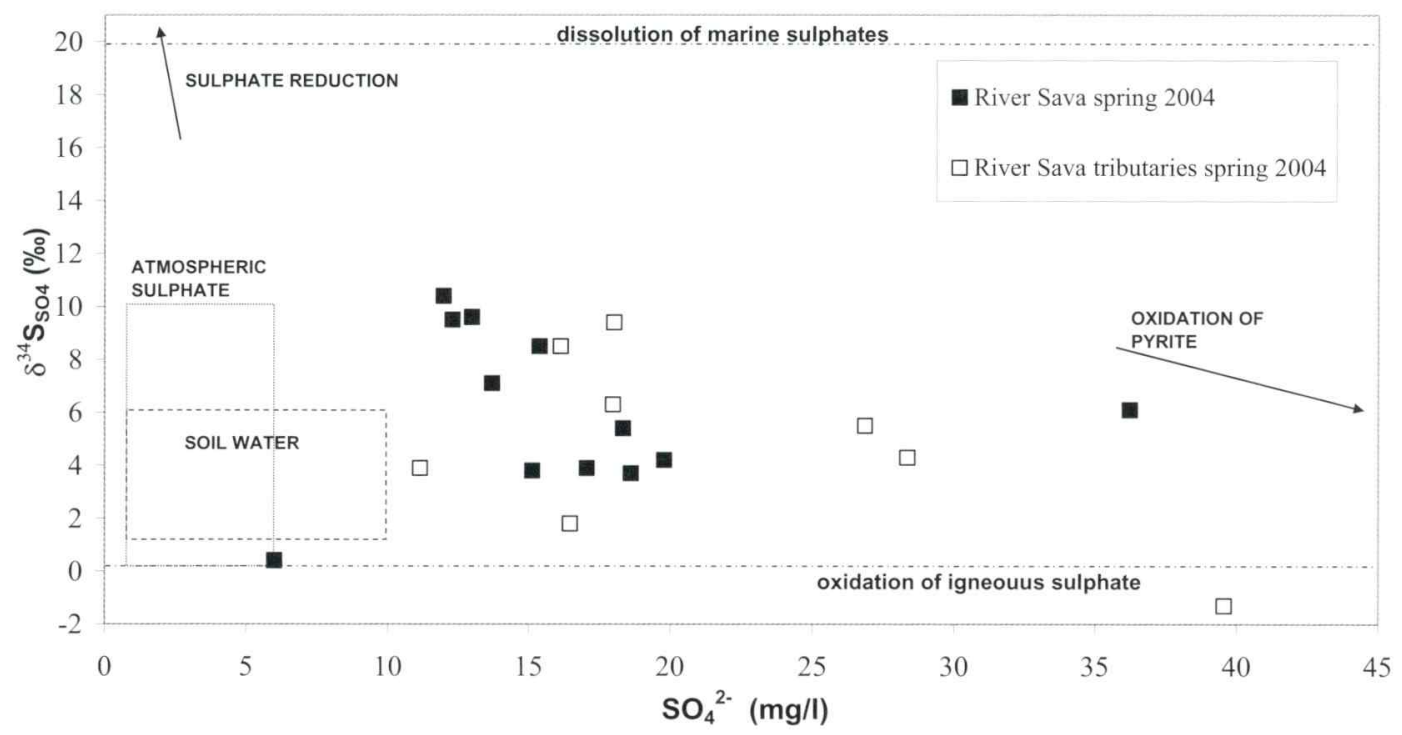

Figure 7. Scatter diagram of sulphur isotopic composition versus sulphate concentration of sulphate for the River Sava system (after Yang et al., 1996).

$\mathrm{Q}_{\mathrm{i}}\left(\mathrm{m}^{3} / \mathrm{s}\right)$ - is discharge in River Sava at the Bregana sampling location (RS) and contributions affecting discharge at this location from tributaries (tri), average annual precipitation amount in the watershed (p) and discharge of other sources (other)

$\left[\mathrm{SO}_{4}{ }^{2-}\right]_{\mathrm{i}}$ - are the measured sulphate concentrations (mmol/l) at the Bregana sampling location (RS) and contributions affecting sulphate concentration at this location from tributaries (tri), precipitation (p) and other sources (other)

$\mathrm{F}_{\text {others }}(\mathrm{mol} / \mathrm{s}), \delta^{34} \mathrm{~S}_{\text {others }}(\%)$ are in equations (4) and (5) unknowns.

Assuming that the sources of $\mathrm{SO}_{4}{ }^{2-}$ to the River Sava arise from the tributaries $\left(\mathrm{F}_{\mathrm{tri}}\right)$, precipitation $\left(\mathrm{F}_{\mathrm{p}}\right)$ and other sources $\left(\mathrm{F}_{\text {other }}\right)$, the contributions of these inputs were calculated to be $52: 8: 40 \%$ for spring 2004 at the Bregana location (41).

The three main tributaries contributing to the sulphate flux are the Tržiška Bistrica $(10.1 \%)$, the Savinja (34\%) and the Krka $(29.3 \%)$. In precipitation a $\delta^{34} \mathrm{~S}_{\mathrm{SO} 4}$ value of $5.2 \%$ was measured in spring 2004. The annual sulphur flow in precipitation according to the average amount of precipitation of $1500 \mathrm{~mm} / \mathrm{a}$ and concentration of sulphate in precipitation $1 \mathrm{mg} / \mathrm{l}$ in spring (Kanduč 2006) is estimated to be $1.7 \times 10^{8}$ mol S/a. The flux from other sources, that accounts for $40 \%$ of $\mathrm{SO}_{4}{ }^{2-}$, may be derived from sources such as dissolution of evaporate minerals, the oxidation of sulphides, or from anthropogenic sources, such as air pollution, smelting of sulphide ores and the chemical industry. Since evaporates and sulphide minerals have a limited distribution in the bed rocks of the Sava channel, and the calculated $\delta^{34} \mathrm{~S}_{\text {other }}$ value is $5.2 \%$, this suggests that evaporates are not the missing contributor. Industrial pollution probably accounts for the bulk of the "other" flux, amounting $8.2 \times 10^{8} \mathrm{~mol} / \mathrm{a}$ of sulphur. Unfortunately, the isotopic composition of sulphur from these industrial operations is not available and this precludes a more precise assessment of industrial impact on the River Sava system. It is known that the Cinkarna Celje factory/industrial complex (sampling location 33) produced 100000 t/a of sulphuric acid from the year 1975 and represents a huge ecological problem for the Celje region (Podpečan, 1988).

From discharge and concentration measurements (EIONET, 2005), the annual sulphate flux was at Bregana (41) was calculated to be $1.5 \times 10^{9} \mathrm{~mol} / \mathrm{a}$. According to Ivanov (1983), the annual $\mathrm{SO}_{4}{ }^{2-}$ flux from continents to oceans is $6.8 \times 10^{12} \mathrm{~mol} / \mathrm{a}$, with contributions from rivers, anthropogenic emissions, and groundwater of 48,48 , and $4 \%$, respectively. 


\section{Conclusion}

In the carbonate part of the Sava watershed lower $\mathrm{Ca}^{2+}$ and $\mathrm{Mg}^{2+}$ concentrations and lower alkalinity were found in comparison to the central and lower part of the river, which is composed of carbonate and clastic rocks. The partial pressure of $\mathrm{CO}_{2}$ ranges from $129 \mathrm{ppm}$ to $9120 \mathrm{ppm}$ and on average is 5 times oversaturated in comparison to atmospheric $\mathrm{CO}_{2}(316 \mathrm{ppm})$. The River Sava and its tributaries represent a source of $\mathrm{CO}_{2}$ to the atmosphere in all sampling seasons, especially in late summer months. Thermodynamic calculations indicate precipitation of calcite and dolomite in the river system, but $\delta^{13} \mathrm{C}_{\mathrm{PIC}}$ values reveal detritical carbonate.

$\delta^{13} \mathrm{C}_{\mathrm{DIC}}$ values change seasonally from 13.5 to $-3.3 \%$ and reflect relations between the terrestrial, riverine and atmospheric reservoirs and the following processes: (1) influence of tributaries, (2) dissolution of carbonates, (3) degradation of organic matter and (4) equilibration with atmospheric $\mathrm{CO}_{2}$. The annual long term flux of dissolved inorganic carbon is estimated to be $4.1 \times 10^{11}$ g C/year, and according to drainage area, $4 \times$ $10^{7} \mathrm{~g} \mathrm{C} /$ year $\mathrm{km}^{2}$ at the border with Croatia.

$\delta^{18} \mathrm{O}_{\mathrm{H} 2 \mathrm{O}}$ values in the River Sava change seasonally from -11.0 to $-8.3 \%$, while in the tributaries they change from -12.4 to $-8.6 \%$. More positive values are attributed to the evaporation process. More negative $\delta^{18} \mathrm{O}_{\mathrm{H} 2 \mathrm{O}}$ values are detected in spring months due to snow melting, while in the winter months $\delta^{18} \mathrm{O}_{\mathrm{H} 2 \mathrm{O}}$ values are comparable to the late summer months.

In the Sava watershed sulphate concentrations change seasonally from 4.34 to $55.5 \mathrm{mg} / \mathrm{l}$. Concentrations of sulphate and $\delta^{34} \mathrm{~S}_{\mathrm{SO} 4}$ values indicate that the source of the Sava Dolinka spring is mainly recharged from precipitation. From discharge data, concentrations of sulphate in water and the drainage area, the sulphate flux is estimated to be $1.4 \times 10^{7} \mathrm{~g} \mathrm{SO}_{4} / \mathrm{km}^{2}$ at the border with Croatia. Assuming that the sources of $\mathrm{SO}_{4}{ }^{2-}$ to the River Sava are its tributaries, precipitation and other sources, the ratio of the contributions of these inputs are calculated to be $52: 8: 40 \%$. Other sources could be attributed to industrial pollution and oxidation of sulphide minerals. The calculated $\delta^{34} \mathrm{~S}_{\mathrm{SO} 4}$ value for other sources is $5.1 \%$, which indicates that the greatest contribution of these other source is from industrial activity.

\section{Acknowledgements}

The authors are thankful to Mr. Stojan Žigon for technical support. This research was conducted in the framework of the project L2-6458-792 funded by the Slovenian Research Agency (ARRS) and within the EU $6^{\text {th }}$ Framework Specific Targeted Research Project - SARIB (Sava River Basin: Sustainable Use, Management and Protection of Resources), Contract No. INCO-CT2004-509160. The project was also financially supported by the National Science Foundation, USA (NSF-EAR\#0208182). Sincere thanks to Anthony Byrne for improving the English of the manuscript.

\section{References}

Agency of Republic of Slovenia for the Environment (ARSO), 2004: monthly bulletin. - No. 1-12. (in Slovene).

Appelo, C. A. J. \& Postma, D. 1994: Geochemistry, groundwater and pollution. - A. A. balkema, 536 pp., Rotterdam/Brookfield.

Atkins, P. W. 1994: Physical chemistry. - Oxford University press, $1031 \mathrm{pp}$., Oxford.

Barth, J. A. C., Cronin, A. A., Dunlop, J., \& Kalin, R. M. 2003: Influence of carbonates on the riverine carbon cycle in an antropogenically dominated catchment basin: evidence from major elements and stable carbon isotopes in the Lagan River (N. Ireland). - Chemical Geology, 200, 203-216.

Buser, S. \& Draksler, V. 1989: Geological composition of Slovenia. In: Javornik, M., Voglar, D., Dermastia, A. (Eds.). Encyclopedia of Slovenia, 3, Mladinska knjiga Ljubljana, 200-201 (in Slovene).

Clark, I. \& Fritz, P. 1997: Environmental Isotopes in Hydrogeology. - Lewis Publishers, 328 pp., New York.

Cruz - San, J., Araguas, L., Rozanski, K., Benavente, J., Cardenal, J., Hidalgo, M. C., Garcia - Lopez, S., Martinez - Garrido, J. C., Moral, F. \& Olias, M. 1992: Sources of precipitation over South-Eastern Spain and groundwater recharge. An isotopic study. - Tellus, $44 \mathrm{~B}$, 226-236.

Dansgaard, W. 1964: Stable isotopes in precipitation. - Tellus, 16, 436-468.

Dever, L., Durand, R., Fontes, J. Ch. \& Vaicher, P. 1983: Etude pédogénétique et isotopique des néoformations de calcite dans un sol sur craie. - Caractéristiques et origines. - Geochim. Cosmochim. Acta, 47, 2079-2090.

Elderfield, H., Upstill-Goddard, R. \& Sholkovitz, E. R. 1990: The rare earth elements in rivers, estuaries and coastal seas and their significance to the composition of ocean waters. - Geochimica Cosmochimica Acta, 54, 971-997.

EIONET (2005) European Environment Information and Observation Network http://eionet-eu.arso.gov.si Cited 11 Nov 2005. 
Fairchild, I. J., Bradby, L., Sharp, M. \& Tison, J.-L. 1994: Hydrogeochemistry of carbonate terrains in alpine glacial setting. Earth Surfaces Processes Landforms, 19, 33-54.

Fairchild, I. J., Killawee, J. A., Hubbard, B. \& Dreybrodt, W. 1999. Ineractions of calcareous suspended sediment with glacial meltwater, a field test of dissolution behaviour. - Chemical geology, 155, 243-263.

Fairchild, I. J., Borsato, A., Tooth, A. F., Frisia, S., Hawkesworth, C. J. Huang, Y., McDermott, F. \& Spiro, B. 2000: Controls on trace element (Sr-Mg) compositions of carbonate cave waters: implications for spelothem climatic records. - Chemical geology, 166, 255-269.

Gaillardet, J., Dupré, B. \& Allègre, C. J. 1999: Global silicate weathering and $\mathrm{CO}_{2}$ consumption rates deduced from the chemistry of large rivers. - Chemical geology, 159, 3-30.

Gao, W. \& Kempe, S. 1987: The Changjiang: its long - term change in $\mathrm{p}_{\mathrm{CO} 2}$ and $\mathrm{p}_{\mathrm{CO} 2}$ carbonate mineral saturation. In: Degens E. T. (Ed.), Transport of Carbon and Minerals in Major World Rivers, Mitt. Geol. - Paläont. Inst. Univ. Hamburg, 64, 207-216.

Gibbs, R. J. 1972. Water chemistry of the Amazon River. - Geochimica Cosmochimica Acta, 36, 1061-1066.

Goldstein, S. J. \& Jacobsen, S. B. 1988: Rare earth element in river waters. - Earth Planetary Science Letters, 89, 35-47.

Gonfiantini, R. 1986: Environmental isotopes in lake studies. In: Fritz, P., Fontes J. Ch. (Eds.) Handbook of Environmental Isotope Geochemistry., 2, 113-168.

Hrvatin, M. 998: Discharge regimes in Slovenia. - Geografski zbornik, XXXVIII, 60-87.

Hu, M.-H., Stallard, R. F. \& Edmond, J. M.. 1982: Major ion chemistry of some large Chinese rivers. - Nature, 298, $550-553$.

Huh, Y., Tsoi, M. Y., Zaitsev, A. \& Edmond, J. M. 1998: The fluvial geochemistry of the rivers of Eastern Siberia: I. Tributaries of the Lena River draining the sedimentary platform of the Siberian Craton. - Geochimica Cosmochimica Acta, 62, 1657-1676.

IAEA 2005: Isotopic composition of precipitation in the Mediterranean Basin in relation to air circulation patterns and climate. Final report of a coordinated research project 2000-2004. IAEA-TECDOC-1453, Vienna : IAEA, 223 pp.

Ivanov, M. V. 1983: The sulfur cycle in continental reservoirs. In: Ivanov M. V., Freney R. J. (Eds.) - The Global Biogeochemical Sulphur Cycle. 19, Wiley, SCOPE 19, 297-356.

Javornik, M., Voglar, D. \& Dermastia A., (Eds.) 1988: Encyclopedia of Slovenia, 2, pp. 68, Mladinska knjiga, Ljubljana.

Kanduč, T. 2006: Hydrogeochemical characteristics and carbon cycling in the River Sava watershed in Slovenia. - Ph. D. Thesis, pp. 141, University of Ljubljana (in Slovene).

Kanduč, T., Szramek, K., Ogrinc, N. \& Walter M. L. 2007: Origin and cycling of riverine inorganic carbon in the Sava River watershed (Slovenia) inferred from major solutes and stable isotopes, Biogeochemistry in press.

Karim, A. \& Veizer, J. 2000: Weathering processes in the Indus River Basin: implications from riverine carbon, sulphur, oxygen, and strontium isotopes. - Chemical geology, 170, 153-177.
Kempe, S. 1982: Long-term record of $\mathrm{CO}_{2}$ pressure fluctuations in fresh waters: In Degens E.T. (Ed.) Transport of Carbon and Minerals in Major World Rivers, Part 1, 52, pp. 91-332. Mitt. Geol.-Paläont. Inst. Univ. Hamburg.

Krouse, H. R. 1980: Sulphur isotopes in our environment. In: Fritz P., Fontes J. Ch. (Eds.) Handbook of Environmental Isotope Geochemistry I The Terrestrial Environment, Elsevier, Amsterdam, 435-472.

Liu, Z. \& Zhao, J. 2000: Contribution of carbonate rock weathering to the atmospheric $\mathrm{CO}_{2}$ sink. - Environmental geology, 39, 10531058 .

Meybeck, M. 1996: River water quality: global ranges, time and space variabilities, proposals for some redefinitions. - Verh. Internat. Verein Limnol., 26, 81-96.

O'Neil, J. R. 1979: Stable Isotope Geochemistry of Rocks and Minerals. - V: Lectures in Isotope Geology, Jager, E., Hunzinger, J. C., (Eds). - Springer Verlag, 235-263, Berlin.

Palmer, S. M., Hope, D., Billett, M. F., Dawson, J. J. \& Bryant, C.L. 2001: Sources of organic and inorganic carbon in a headwater stream: evidence from carbon isotope studies. - Biogeochemistry, 52, 321-338.

Parkhurst, D. L. \& Appelo, C. A. J. 1999: User's guide to PHREEQC for Windows (version 2) - a computer program for speciation, batch reaction, one - dimensional transport, and inverse geochemical calculations. - Water - Resources Investigations Report, 99-4259.

Pawellek, F., Frauenstein, F., \& Veizer, J. 2002: Hydrochemistry and isotope geochemistry of the upper Danube River. - Geochimica et Cosmochimica Acta, 66/21, 3839-2854.

Pearson, F. J. \& Rightmire, C. T. 1980: Sulphur and oxygen isotopes in aqueous sulphur compounds. In: Fritz P. and Fontes J. Ch. (Eds.) Handbook of Environmental Isotope Geochemistry, 1, 227-259, Elsevier.

Petkovšek, Z. 1969: Fog occurrence in lowlands of Slovenia, Thesis. - Društvo meteorologov Slovenije, 11, 57-89 (in Slovene).

Podpečan, D. 1988: Cinkarna Celje. In: Javornik M., Voglar D., Dermastia A., (Eds.) Encyclopedia of Slovenia, 2, Mladinska knjiga, Ljubljana, pp. 68 (in Slovene).

Ramovš, A. 1983: Waterfalls in Slovenia. - Slovenska matica, 1983, 188 pp., Ljubljana.

Reeder, S. W., Hitchon, B. \& Levinson, A. A. 1972: Hydrogeochemistry of the surface waters of the Mackenzie River drainage basin, Canada: 1. Factors controlling inorganic composition. - Geochimica Cosmochimica Acta, 36, 181-192.

Stallard, R. F. \& Edmond, J. M. 1983: Geochemistry of the Amazon: 2. The influence of geology and weathering environment on the dissolved load. - Journal of Geophysical Research, 88, 9671-9688.

Telmer, K. \& Veizer, J., 1999: Carbon fluxes, $\mathrm{pCO}_{2}$ and substrate weathering in a large northern river basin, Canada: carbon isotope perspectives. - Chemical geology, 159, 61-86.

Urbanc, J. \& Brancelj, A. 2002: Hydrological Connections between some lakes in the Triglav Lakes Valley. In: Brancelj A. (Ed.) High - mountain lakes in the eastern part of the Julian Alps., Ljubljana : ZRC SAZU, National Institute for biology, pp. 77-90. 
Vreča, P., Kanduč, T., Žigon, S. \& Trkov, Z. 2004: Isotopic composition of precipitation in Slovenia. In: Isotopic composition of precipitation in the Mediterranean basin in relation to air circulation patterns and climate: final report of a coordinated research project 2000-2004, (IAEA-TECDOC, 1453). - Vienna: IAEA, 2004, pp. $157-172$.

Yang, C., Telmer, K., \& Veizer, J. 1996: Chemical dynamics of the 'St. Lawrence' riverine system: $\delta \mathrm{D}_{\mathrm{H} 2 \mathrm{O}}, \delta^{18} \mathrm{O}_{\mathrm{H} 2 \mathrm{O}}, \delta^{13} \mathrm{C}_{\mathrm{DIC}}, \delta^{34}$ Ssulfate and dissolved ${ }^{87} \mathrm{Sr} /{ }^{86} \mathrm{Sr}$. - Geochimica Cosmochimica Acta, 60, 851-866.
Yee, P., Edgett, R., \& Eberhardt, A. 1990: Great lakes - St. Lawrence River regulation; what it means and how it works. Joint publication of Environment Canada Ontario Region, and the U. S. Army Corps of Engineers.

Zhang, J., Quay, P. D. \& Wilbur, D. O. 1995: Carbon isotope fractionation during gas - water exchange and dissolution of $\mathrm{CO}_{2}$. - Geochimica Cosmochimica Acta, 59/1, 107-1146.

Zupančič, B. 1998: Precipitations. - Geografski atlas Slovenije, DZS, 99 pp., Ljubljana. 